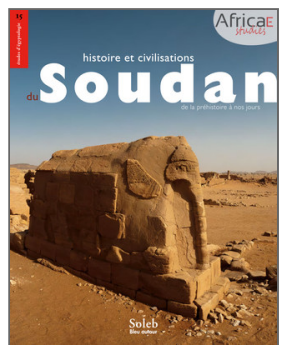

Olivier Cabon (dir.)

Histoire et civilisation du Soudan

De la préhistoire à nos jours

\title{
8. Hiéroglyphes africains
}

À la recherche de la langue perdue de Méroé

\section{Claude Rilly}

DOI : 10.4000/books.africae.2822

Éditeur: Africae, Soleb, Bleu autour

Lieu d'édition : Paris, Khartoum

Année d'édition : 2017

Date de mise en ligne : 17 janvier 2022

Collection : Africae Studies

EAN électronique : 9782493207074

\section{Q OpenEdition}

\section{Books}

http://books.openedition.org

\section{Référence électronique}

RILLY, Claude. 8. Hiéroglyphes africains : À la recherche de la langue perdue de Méroé In : Histoire et civilisation du Soudan : De la préhistoire à nos jours [en ligne]. Paris, Khartoum : Africae, 2017 (généré le 28 janvier 2022). Disponible sur Internet : <http://books.openedition.org/africae/2822>. ISBN : 9782493207074. DOI : https://doi.org/10.4000/books.africae.2822. 


\title{
hiéroglyphes africains
}

\author{
à la recherche
}

de la langue perdue de Méroé 
Parmi tous les mystères dont le royaume de Méroé est nimbé, sa langue est sans doute le plus tenace. Les textes existent, en grand nombre, mais beaucoup d'entre eux - et surtout les plus riches en informations, les chroniques royales - résistent en grande partie à la traduction depuis plus d'un siècle. Il est faux, comme on le lit parfois, que les inscriptions méroïtiques ne soient pas déchiffrées. L'écriture est en effet connue depuis les travaux de Griffith, entre 1907 et 1911, et seule la valeur de quelques rares signes (des unités de mesure) nous échappe. Le méroïtique appartient à ce petit groupe de langues anciennes, d'origine et de localisation diverses, dont l'écriture est connue mais la langue en grande partie ignorée. Il y voisine avec l'étrusque, le gaulois, le picte d'Écosse, le messapien de la région des Pouilles en Italie, etc. Toutes ces langues peuvent donc être lues, au sens le plus strict du terme, car la valeur des signes est connue, mais la signification des mots nous échappe en grande partie, si bien qu'il est impossible de traduire l'ensemble des textes.

Il est également faux que le méroïtique soit une langue totalement obscure. On connaît un peu de vocabulaire et des pans non négligeables de la grammaire. La plupart des textes funéraires, qui constituent presque le tiers du corpus actuel, sont aujourd'hui bien compris. On sait même depuis quelques années à quelle famille linguistique appartenait le méroïtique, alors que cette énigme avait défié les chercheurs pendant un siècle. Malheureusement, aucune des langues apparentées n'est assez proche pour assurer une traduction aisée. Si le français était un idiome oublié, la connaissance de l'italien permettrait de comprendre assez vite des textes écrits en cette langue, mais la connaissance du russe, pourtant classé dans la même famille indo-européenne, serait d'un piètre secours pour le même exercice. C'est que, si la séparation de l'italien et du français s'est faite il y a un millénaire et demi, celle de l'italique (dont descendra le latin, puis le français) et du slave (dont descendra le russe) remonte sans doute à près de quatre mille ans. Le groupe de langues le plus proche génétiquement du méroïtique, le nubien, s'en est séparé depuis à peu près le même espace de temps, ce qui explique leurs importantes différences.

\section{Le déchiffrement de l'écriture méroïtique une conquête britannique oubliée}

En 2010 eut lieu à Londres, au British Museum, la douzième conférence internationale des Études nubiennes. Assez curieusement, les Britanniques, qui ont en général un sentiment patriotique bien affirmé, laissèrent passer l'occasion de célébrer le centenaire du déchiffrement de l'écriture 
le Soudan

\section{2}

des origines

à la chute

du sultanat

Fung méroïtique par leur compatriote Francis Ll. Griffith. Pourtant, comme nous allons le voir, cette découverte n'était pas un mince exploit, d'autres savants s'y étant essayés sans succès pendant près d'un demi-siècle. S'il a existé un "Champollion du méroïtique», c'est bien Griffith. Certes, il n'a pu, à l'aide de cette clef retrouvée, donner immédiatement accès à l'ensemble des textes, contrairement à son glorieux collègue français. Mais cette différence tient à la destinée des deux langues: l'égyptien avait subsisté sous la forme du copte, alors que le méroïtique avait disparu sans descendance. D'autre part, si des textes bilingues, comme la fameuse Pierre de Rosette, permettaient d'avoir une version grecque d'un texte égyptien, aucune inscription importante de ce type, juxtaposant au méroïtique soit le grec, soit l'égyptien, n'a été jusqu'à présent retrouvée. C'est ce qui rend d'autant plus admirable la prouesse de Griffith.

Contrairement à ce qui s'était passé en Égypte, on ne se souvenait même plus qu'il avait existé une langue et une écriture originales au temps de ces rois dont les pyramides se dressaient encore à Méroé. Il fallut attendre les voyages de quelques hardis explorateurs pour que le monde savant redécouvrît le méroïtique. Le premier relevé d'inscription fut effectué par l'architecte franco-allemand Franz Christian Gau en 1819 dans le temple de Dakka et apparut sans commentaires sur une planche de son ouvrage, Antiquités de la Nubie ou Monuments inédits des bords $d u$ Nil, entre la première et la seconde cataracte, publié en 1822. C'était l'année où Champollion déchiffrait les hiéroglyphes, et nul, pas même l'auteur de la Lettre à M. Dacier, n'accorda d'attention à cette ligne de signes incompréhensibles.

La même année parut à Londres, sous la plume des voyageurs britanniques George Waddington et Barnard Hanbury, un livre intitulé Journal of a Visit to Some Parts of Ethiopia, où figurait le relevé d'un graffito en cursive méroïtique de la salle hypostyle du temple de Soleb. Un commentaire laconique définissait l'inscription comme écrite «en caractères inconnus de nous, bien que ressemblant fortement à du grec». Dans les mêmes années (1820-1822) eut lieu la conquête du Soudan par Ismaïl Pacha. Suivant de près l'armée turco-égyptienne du prince, le Français Frédéric Cailliaud visita un grand nombre de sites mérö̈tiques et copia cinq inscriptions qui parurent en 1826 dans le troisième tome de sa relation de voyage, Voyages à Méroé, au Fleuve Blanc, au-delà du Fâzoql, dans le midi du royaume de Sennâr, à Sywah et dans cinq autres oasis, faits dans les années 1819, 1820, 1821 et 1822. Il fut d'ailleurs le premier à reconnaître l'originalité de cette écriture ("caractères éthiopiens", c'està-dire, à l'époque, "soudanais"), mais cette assertion reposait sur l'intuition, puisque les études égyptologiques étaient alors dans leur enfance. 
Ni Champollion, ni Rosellini, qui passèrent à Kalabcha et à Philae lors de leur expédition franco-toscane en 1828 , n'accordèrent d'importance aux quelques textes méroïtiques inscrits sur les murs de ces temples: ils avaient déjà suffisamment de travail avec l'égyptien.

C'est avec le grand égyptologue allemand Richard Lepsius que fut enfin reconnue la civilisation de Méroé. Son œuvre prodigieuse, les Denkmäler aus Aegypten undAethiopien [Monuments d'Égypteet d'Ethiopie], est le fruit d'une expédition de trois ans, de 1842 à 1845 , le long de la vallée du Nil. L'ouvrage parut de 1849 à 1858 . Les volumes V et VI, consacrés à la Nubie, comportaient, outre de nombreuses planches détaillant les monuments, cinquantetrois relevés d'inscriptions, qui allaient permettre à deux générations de savants d'exercer leur sagacité. Parmi les objets qu'il rapporta à Berlin figurait un grand reposoir de barque sacrée retrouvé à Ouad Ben Naga, non loin de Méroé, et qui avait la particularité de présenter les noms de deux monarques, la reine Amanitoré et le roi Natakamani, dans des cartouches rédigés en hiéroglyphes égyptiens et en hiéroglyphes méroïtiques. Ce monument allait dans les années qui suivirent jouer un grand rôle dans les tentatives de déchiffrement. Lepsius avança déjà un certain nombre d'observations et d'hypothèses dont plusieurs frappent par leur clairvoyance: 《 $\mathbf{D}$ 'un usage plus courant et commun que les hiéroglyphes, existait en ce temps une écriture démotique éthiopienne [la cursive méroïtique], dont les tracés ressemblent au démotique égyptien, bien qu'elle utilise un alphabet au nombre de signes très réduit, consistant en 25 à 30 caractères. Cette écriture se lit pareillement de droite à gauche, mais les mots y sont séparés de manière constante par deux points marqués. [...] Le déchiffrement de cette écriture, au terme d'une étude précise, ne sera peut-être pas difficile et devrait alors nous livrer les premiers sons de la langue éthiopienne parlée ici. \\(Lepsius 1952: Briefe aus Ägypten, Äthiopien und der Halbinsel des Sinai. Lettre du 22 avril 1844, écrite des pyramides de Méroé).

En revanche, un certain nombre de suppositions devaient se révéler fausses: ainsi Lepsius n'était-il pas convaincu en 1844 que l'écriture hiéroglyphique fût autre chose qu'une imitation des hiéroglyphes égyptiens à but uniquement décoratif. Il croyait également que le vieuxnubien, la langue des royaumes chrétiens médiévaux du Soudan, dont il avait pu observer quelques inscriptions à Soba et à Musawwarat, était au méroïtique ce que le copte était à l'égyptien, et il affirma d'ailleurs dans un premier temps que la langue de Koush était l'ancêtre du nubien moderne. Aussi consacra-t-il beaucoup de temps à étudier cette langue, dont il publia une des premières grammaires quelques décennies plus tard. Curieusement, une autre hypothèse de filiation apparaît dès 1844 dans une lettre datée du 24 novembre, donc peu de temps après la première: 
le Soudan

\section{4}

des origines

à la chute

du sultanat

Fung les Bedjas, peuple nomade vivant le long de la mer Rouge, seraient les descendants des Méroïtes. En 1880, dans la préface de sa Nubische Grammatik, il revint à cette dernière opinion et soutint plus clairement que la langue bedja avait dû être celle de l'Empire de Méroé. Malheureusement il n'en donna aucune justification, bien qu'il soit possible que ce changement d'opinion ait été dû à une recherche jamais publiée sur les inscriptions qu'il avait recueillies. Malgré ces quelques approximations, on peut assurément saluer en Lepsius le génial précurseur du déchiffrement de l'écriture méroïtique. Tous ceux qui lui succédèrent dans cette étude, Griffith inclus, utilisèrent avec profit ses relevés et ses observations.

La prédiction de Lepsius, selon laquelle le déchiffrement ne serait peut-être pas difficile, ne se réalisa pas tout de suite. Plusieurs grands noms de l'égyptologie ou de l'orientalisme s'y essayèrent en vain pendant des décennies. Derrière les apparences policées des échanges savants, on devine combien était vive la concurrence entre les chercheurs pour savoir qui apporterait à son pays la gloire d'un second déchiffrement, après celui des hiéroglyphes égyptiens que Champollion avait mené victorieusement pour l'honneur de la France. Une première tentative, à partir des noms royaux, fut conduite par l'Anglais Samuel Birch en 1868. Mais une mauvaise lecture des hiéroglyphes égyptiens, de graphie particulière, contenus dans le cartouche du roi Natakamani sur le reposoir de barque de Ouad Ben Naga, ainsi qu'un rapprochement erroné avec l'amharique, langue principale de l'actuelle Éthiopie, l'empêchèrent d'aller plus avant.

Après lui, le grand démotisant Heinrich Brugsch publia le résultat de ses recherches sur le sujet en deux articles qui parurent en 1887 dans la même livraison du Zeitschrift für ägyptische Sprache, dont il était le rédacteur, sous le titre encore trop ambitieux d'Entzifferung der meroitischen Schriftdenkmäler [Déchiffrement des inscriptions mérö̈tiques]. Un bon nombre de ses hypothèses étaient pourtant d'une grande justesse. Il comprit, contrairement à Birch, que certains hiéroglyphes s'écartaient du modèle égyptien et étaient eux-mêmes sujets à de fortes variations graphiques. Il réduisit en conséquence à 23 le nombre des signes hiéroglyphiques méroïtiques, ce qui correspondait à leur décompte exact, même s'il y arrivait par un ajout et un oubli. Il prédit justement que le nombre des signes cursifs devait être équivalent. Il donna une translittération correcte de douze des hiéroglyphes, et repéra l'article $-l$. En revanche, il crut que le sens de lecture des signes était le même qu'en égyptien, c'est-à-dire que les êtres animés regardaient vers le début de la ligne. Il limita le champ de ses investigations à l'écriture hiéroglyphique, ce qui ne représentait qu'un petit nombre d'inscriptions, souvent lacunaires. Enfin, reprenant 
les premières théories de son maître Lepsius, il s'efforça de retrouver dans le nubien moderne les traces du méroïtique, ce qui ne pouvait que l'entraîner dans une impasse. Au terme de cette étude liminaire, il annonça la parution imminente d'un ouvrage consacré à ce déchiffrement, qui inclurait l'examen de la cursive. Il est fort possible que ce n'ait été qu'une stratégie destinée à décourager les autres chercheurs, comme Reinisch et Dümichen, qui avaient commencé à travailler dans ce domaine. Certains points de la démonstration de Brugsch montrent en effet qu'il ne s'était pas encore intéressé à la cursive. Quoi qu'il en soit, l'ouvrage ne parut jamais et les articles de 1887 ne connurent pas de suite, pas plus d'ailleurs que les recherches de ses rivaux.

Dix ans plus tard, Adolf Erman reprit les travaux de Brugsch, et proposa dans un article de 1897 quelques équivalences supplémentaires. Certaines étaient judicieuses, mais d'autres constituaient un recul par rapport à Brugsch, puisque Erman introduisait dans l'écriture méroïtique des déterminatifs, signes idéographiques connus en égyptien pour encoder la catégorie sémantique des mots. Il ne s'engagea pas, contrairement à son prédécesseur, dans une discussion des rapports du méroïtique avec le nubien, mais proposa très justement de voir dans les transcriptions des noms royaux de la XXVe dynastie les précurseurs de l'écriture hiéroglyphique méroïtique.

La fin de l'insurrection mahdiste au Soudan et l'établissement d'un pouvoir colonial britannique avaient entre-temps permis la reprise des activités archéologiques. Les fouilles de John Garstang à Méroé mirent au jour un certain nombre d'inscriptions qui venaient s'ajouter à celles que Lepsius avait reproduites dans ses Denkmäler. En Basse-Nubie, l'archéologue David Randall-MacIver découvrit à Shablul, puis à Karanóg, plus d'une centaine de textes funéraires. Tout ce nouveau matériel exigeait un spécialiste. Or, peu de temps auparavant, le grand orientaliste Archibald H. Sayce, qui avait déjà avec quelque succès étudié les inscriptions hittites et hourrites, commença à s'intéresser à l'écriture méroïtique et travailla dans la lignée d'Erman. Comme l'article d'Erman, la contribution de Sayce constituait plutôt un recul par rapport aux articles de Brugsch. Le savant y multipliait par deux le nombre des hiéroglyphes. Bien qu'il eût admis le principe du sens de lecture opposé à l'égyptien, exposé par Griffith dans Areika dès 1909, il n'offrait de transcription correcte que pour huit signes, et approximative pour cinq autres. Surtout, il n'était toujours pas question des inscriptions en cursive, qui auraient pourtant permis de multiplier le nombre des équivalences. Il eut la malchance que le résultat de ses recherches non seulement coïncidât avec la publication des premières lectures de Griffith, mais parût, comble d'infortune, dans le même ouvrage édité par Garstang en 1911, Meroë, the City of the Ethiopians. 
Bien qu'il n'eût pas encore atteint les plus hauts rangs de l'université, Francis Llewellyn Griffith (1862-1934) était sans conteste à cette époque le plus brillant philologue de Grande-Bretagne dans le domaine égyptologique. Ses travaux sur le démotique et l'hiératique anormal avaient fait de lui en quelques années l'un des meilleurs spécialistes mondiaux des écritures égyptiennes tardives. Randall McIver lui confia dès 1907 la publication des premiers textes découverts à Shablul lors de son expédition en Nubie. Bientôt, les fouilles de Karanóg livrèrent une abondante moisson de stèles et de tables d'offrandes, qui, ajoutées à celles de Shablul, doublèrent en quelques mois le nombre d'inscriptions méroïtiques connues. En 1909 parut Areika, le premier volume détaillant les nouvelles découvertes effectuées en Nubie. Dans le chapitre Ix, intitulé Meroitic Inscriptions, Griffith exposait les premiers résultats de ses recherches. Il y récapitulait les analyses de ses prédécesseurs et surtout y donnait déjà, avec une amorce de paléographie, la liste exacte des signes cursifs. L'inventaire des hiéroglyphes n'était pas encore au point et comportait huit signes surnuméraires, mais quinze équivalences, toutes justes, entre les écritures cursive et hiéroglyphique y étaient déjà indiquées. Le sens de lecture des hiéroglyphes, inverse de celui des Égyptiens, était clairement défini et constituait à lui seul une avancée décisive. Le chapitre se terminait par un exposé des parentés possibles du méroïtique, où Griffith rejetait, pour des raisons tant philologiques qu'historiques, la filiation du nubien avec la langue de Koush. Les douze pages de ce court exposé levaient tous les obstacles qui avaient jusque-là empêché les progrès: le sens de lecture des hiéroglyphes, l'absence d'étude de la cursive et la fixation sur un hypothétique héritage nubien.

L'Egypt Exploration Fund ne s'y trompa pas, qui misa à son tour

des origines

à la chute

du sultanat

Fung sur Griffith, et lui permit en 1909 de visiter les riches collections berlinoises ramenées par Lepsius, puis de partir copier sur place, en Égypte et au Soudan, toutes les inscriptions disponibles. Ces relevés, étudiés et publiés en un temps record, devaient fournir la matière de deux volumes intitulés Meroitic Inscriptions. Enfin, en 1910, John Garstang mit entre ses mains pour publication les photographies et les relevés des textes découverts durant ses fouilles à Méroé. L'étonnante acuité d'esprit et l'impressionnante puissance de travail de Griffith lui permirent d'avancer rapidement et de mener de front toutes ces lourdes tâches. La publication des inscriptions de Méroé était sous presse en octobre 1910. Les Meroitic Inscriptions I et Karanóg furent envoyés à l'imprimeur en novembre 1910, les épreuves étant révisées et enrichies entre le printemps et l'automne de l'année suivante. Dès le début de cette année 1911 parut dans la revue allemande Zeitschrift für ägyptische Sprache, qui avait déjà accueilli les tentatives de déchiffrement de Brugsch et de Erman, un bref article de deux pages: 
A Meroitic funerary text in hieroglyphic, où Griffith démontrait qu'il «tenait l'affaire", pour reprendre la célèbre formule de Champollion. Le savant britannique donnait en effet une analyse de la table d'offrandes de Takideamani (Rem oo6o), le seul exemple alors connu d'épitaphe en écriture hiéroglyphique, rapporté à Berlin par l'expédition prussienne. L'article débutait par un hommage aux travaux de Lepsius, suivi - in cauda venenum - de quelques lignes où Griffith s'étonnait qu'une pièce aussi capitale pour le déchiffrement n'eût pas été publiée dans les Denkmäler. Suivait une reproduction du texte hiéroglyphique, puis une transcription en cursive qui n'avait d'autre utilité que de montrer l'ampleur des avancées de Griffith sur les deux formes de l'écriture méroïtique. Enfin, une brève analyse du contenu, donnant le nom des divinités invoquées, celui du défunt et de ses parents, prouvait, si besoin en était, que le déchiffrement était complet, et que le travail sur le lexique était déjà bien entamé.

Effectivement, l'année 1911 vit la publication des trois volumes en préparation: Karanóg, les Meroitic Inscriptions I et le chapitre de Meroë consacré à la transcription des textes méroïtiques. Cette dernière contribution, écrite rapidement, n'offrait que peu d'études approfondies des textes et fut surtout pour Griffith l'occasion de prouver la justesse de son déchiffrement. De leur côté, les Meroitic Inscriptions I, qui avaient bénéficié d'un plus long mûrissement, livraient de nombreuses analyses, notamment lexicales, où Griffith, revenu de ses premières théories, proposait divers parallèles nubiens. Mais c'est dans Karanóg, le plus achevé des trois volumes et le premier à paraître, qu'il déployait toute la mesure de son génie. Les trois chapitres d'introduction constituent en effet une synthèse sur la langue et l'écriture méroïtique à laquelle, aujourd'hui encore, il n'y a rien ou presque à changer. Les données qui lui avaient permis le déchiffrement sont tout d'abord exposées avec une grande clarté en une série d'équivalences avec l'égyptien, le copte et le grec. Le deuxième chapitre, consacré à l'étude de la paléographie, définit les trois styles de cursive (archaïque, transitionnel, tardif), en un classement qui fait toujours autorité. Le troisième chapitre résume les données phonologiques, lexicales et grammaticales que Griffith avait réunies en quatre ans de labeur acharné. Là encore, il y a peu à rectifier, et ces quelques pages constituent toujours le noyau de notre connaissance - partielle - des structures de la langue. La majeure partie de l'ouvrage est constituée d'une lecture des textes funéraires de Karanóg et de Shablul, et d'une étude serrée des éléments récurrents dont ils sont composés: invocation, nomination et description du défunt, bénédictions finales. L'ouvrage se termine par l'Index $C$ qui énumère quelque six cents mots et expressions, parfois traduits, et qui reste une référence capitale pour l'étude du lexique méroïtique. 
le Soudan

\section{8}

des origines

à la chute

du sultanat

Fung
L'année suivante parut le second tome des Meroitic Inscriptions, comportant les textes originaires de la région entre Napata et Philae. Cet ouvrage, plus abouti encore que le précédent, offrait des analyses prometteuses et se terminait par un index de huit cents séquences méroïtiques.

Griffith, bien que lucide sur l'ampleur de la tâche qui restait à accomplir, se montrait cependant optimiste pour la suite: 《Tout cela en est encore à un stade initial. Même dans l'alphabet, les voyelles restent extrêmement obscures et, parmi les consonnes, la valeur attribuée à la lettre est sans doute encore incertaine; pour ce qui est du vocabulaire méroïtique, à l'exception des noms de personnes et de lieux et des termes empruntés à l'égyptien, presque rien n'est connu. Mais on peut espérer que le matériel présenté ici a été vérifié, classé et étudié, au point qu'une étincelle supplémentaire suffirait à y apporter promptement toute la lumière. Si des yeux neufs, ceux de déchiffreurs aguerris ou de savants experts en langues d'Afrique du Nord, voulaient s'y exercer, les secrets du méroïtique devraient bientôt être percés. \》(Griffith 1911, Karanóg, Preface, p. vI)

Dans les années qui suivirent, Griffith travailla sans relâche à la traduction du méroïtique. Il s'attela notamment à l'étude du vieuxnubien, la langue écrite des royaumes chrétiens médiévaux, et publia en 1913 The Nubian Texts of the Christian Period, œuvre qui devait rester pendant quinze ans la meilleure synthèse de cette langue. Il lui fallut cependant admettre que ce n'était pas là la clef du problème, car les deux idiomes différaient trop. Les résultats de ses travaux sur le méroïtique proprement dit parurent en quatre articles intitulés Meroitic Studies dans la revue britannique Journal of Egyptian Archaeology en 1916 et 1917. Une certaine amertume les marquait, Griffith se désolant que les progrès vers la traduction soient si lents, mais ces quelques pages permettaient de nouvelles avancées dans l'étude des chiffres, de la phonologie, des structures verbales et du contenu des stèles royales. Cependant, la carrière brillante de Griffith continuait. Il devait mener de front un enseignement universitaire au Queen's College d'Oxford, une activité éditoriale intense, la rédaction d'innombrables articles et rapports, ainsi que plusieurs directions de fouilles en Égypte et au Soudan. Cette besogne incessante, ajoutée à sa déconvenue face à la lenteur des progrès, explique qu'il ait si peu publié sur le méroïtique après la Première Guerre mondiale, se limitant à quelques articles dans le Journal of Egyptian Archaeology.

Certains témoignages, comme celui-ci, rapporté par Bryan Haycock en 1978, font amèrement regretter qu'il n'ait pu poursuivre une tâche qu'il avait si brillamment débutée: 《KGriffith pensait que, s'il pouvait se concentrer seulement sur le méroïtique pour cinq ans de plus, il en savait assez pour résoudre la plupart des problèmes qui s'y posaient, 
mais il lui fallait consacrer beaucoup de temps à organiser et à financer son projet favori, les fouilles d'Oxford en Nubie à Sanam, Faras et Kawa, ainsi qu’à s'occuper de sujets purement égyptologiques, puisqu'il était reconnu comme le principal expert de sa génération sur l'hiératique et le démotique, et des collègues, pensant bien faire, accumulaient sur lui des masses de textes nouveaux, sans comprendre l'importance bien plus grande de ses travaux sur le méroïtique.

\section{Les études méroïtiques de Griffith à nos jours}

Entre la fin des travaux de Griffith et le milieu des années 1950, les études méroïtiques ne suscitèrent plus grand intérêt. Seuls sont à noter la publication des graffiti du temple de Kawa par M. F. L. Macadam, disciple de Griffith, et les travaux très contestables d'un linguiste autrichien, Ernst Zyhlarz. Depuis l'échec de la comparaison avec les langues nubiennes, l'avis général était que le méroïtique appartenait à la famille afro-asiatique (dite aussi chamitosémitique), comme l'égyptien ancien, les langues berbères et sémitiques et celles de la Corne de l'Afrique, aujourd'hui appelées couchitiques (bedja, afar, somali, etc.). C'est plus spécifiquement à ce dernier rameau que l'on reliait la langue de Koush et cette hypothèse explique le nom de "couchitique» (tiré de «Koush») toujours utilisé pour cette famille, bien que l'on sache maintenant que cette filiation est inexacte. C'est à partir de cette parenté supposée que Zyhlarz étudia le méroïtique, n’hésitant pas à distordre les données pour les faire coïncider avec les parlers couchitiques. C'est ainsi qu'il proposa de distinguer un genre grammatical en méroïtique, alors que Griffith avait démontré depuis longtemps qu'il n'en existait pas.

En 1955, un nouveau venu sur ce terrain, Fritz Hintze (1915-1993), professeur d'égyptologie à l'université Humboldt de Berlin-Est, fit paraître une réfutation des thèses de Zyhlarz, intitulée Die Sprachliche Stellung des Meroitischen [La position linguistique du méroïtique]. Dans ce premier travail où se manifestait déjà une grande maîtrise du sujet, Hintze prouvait l'absence de genre en méroïtique, analysait les formes verbales des bénédictions finales des textes funéraires et affirmait l'originalité de la langue, isolée selon lui parmi les grandes familles linguistiques africaines. Il ne devait jamais démordre de cette opinion, attaquant systématiquement les affiliations diverses que l'on proposa par la suite. Puisqu'on ne pouvait selon lui espérer quelque progrès de la méthode comparative, Hintze privilégia l’analyse interne de la langue. En 1963 parut dans les Mitteilungen des Instituts für Orientforschung un essai de trente pages: Die Struktur der "Deskriptionssätze" in den meroitischen Totentexten [La structure des "phrases de description" dans les textes funéraires méroïtiques] qui demeura durant quarante ans 
un bréviaire pour les méroïtisants. L'article traitait en effet des propositions qui indiquent dans les épitaphes la position sociale du défunt, et les classait selon la présence d'un nom propre, d'un génitif, d'un locatif, d'un terme de parenté et de leurs combinaisons. Si les principes syntaxiques exposés par Griffith n'étaient pas fondamentalement améliorés, la présentation des données fournissait aux chercheurs une noria d'exemples dûment translittérés, vérifiés et augmentés de textes découverts peu auparavant. Une liste de mots transcrits en caractères romans, donc beaucoup plus accessible et maniable que les index en écriture méroïtique de Griffith et de Macadam, figurait en annexe. Par la suite, Hintze publia de nombreux articles et contributions orientés dans le même sens, la recherche des structures syntaxiques. Il fut également le premier à démontrer que l'écriture méroïtique n'était pas un "alphabet», fût-il défectif comme le supposait Griffith, mais un alphasyllabaire semblable dans ses principes aux écritures indiennes, un point sur lequel nous reviendrons. Toutefois, l'apport des travaux de Hintze sur le plan sémantique, c'est-à-dire vers une traduction des textes, était décevant. Ses méthodes furent reprises, de manière moins rigide, par Inge Hofmann, professeur à l'université de Vienne, qui privilégia une approche multicontextuelle, conjuguant dans ses études de textes méroïtiques l'analyse structurelle héritée de Hintze et celle du contexte archéologique, historique et culturel des inscriptions. Elle publia entre 1967 et 1993 plus d'une soixantaine d'articles et de monographies consacrées à la culture et à la langue méroïtiques, dont un petit volume paru en 1981 et intitulé Material für eine meroitische Grammatik [Matériel pour une grammaire méroïtique], qui reste encore aujourd'hui une référence en ce domaine.

des origines

à la chute

du sultanat

Fung

Avec la campagne de sauvetage des monuments de Nubie lancée par l'Unesco en 1960 et qui dura jusqu'à l'ennoiement de la région sous les eaux du lac de retenue du barrage d'Assouan en 1964, les études méroïtiques, devenues depuis la mort de Griffith une spécialité germanique, prirent une dimension internationale. De nombreux sites de Nubie furent en effet fouillés et plusieurs livrèrent des inscriptions méroïtiques en grand nombre, suscitant l'intérêt de nouveaux chercheurs. On citera ainsi le Canadien Bruce Trigger et l'Américain Nicholas B. Millet, l'Espagnol Martin Almagro, les Russes Isidor Katznelson et Youri Zawadowski, le Soudanais Abdelgadir Mahmoud Abdalla. En France, une entreprise de longue haleine, le catalogage complet de tous les textes méroïtiques, publiés jusque-là dans des monographies et articles dispersés, fut lancé en 1959 par le professeur Jean Leclant et André Heyler, sous le nom de Répertoire d'épigraphie méroitique (en abrégé REM). Ce projet bénéficia, ce qui était une première dans l'étude des langues anciennes, du secours des techniques informatiques alors naissantes. 


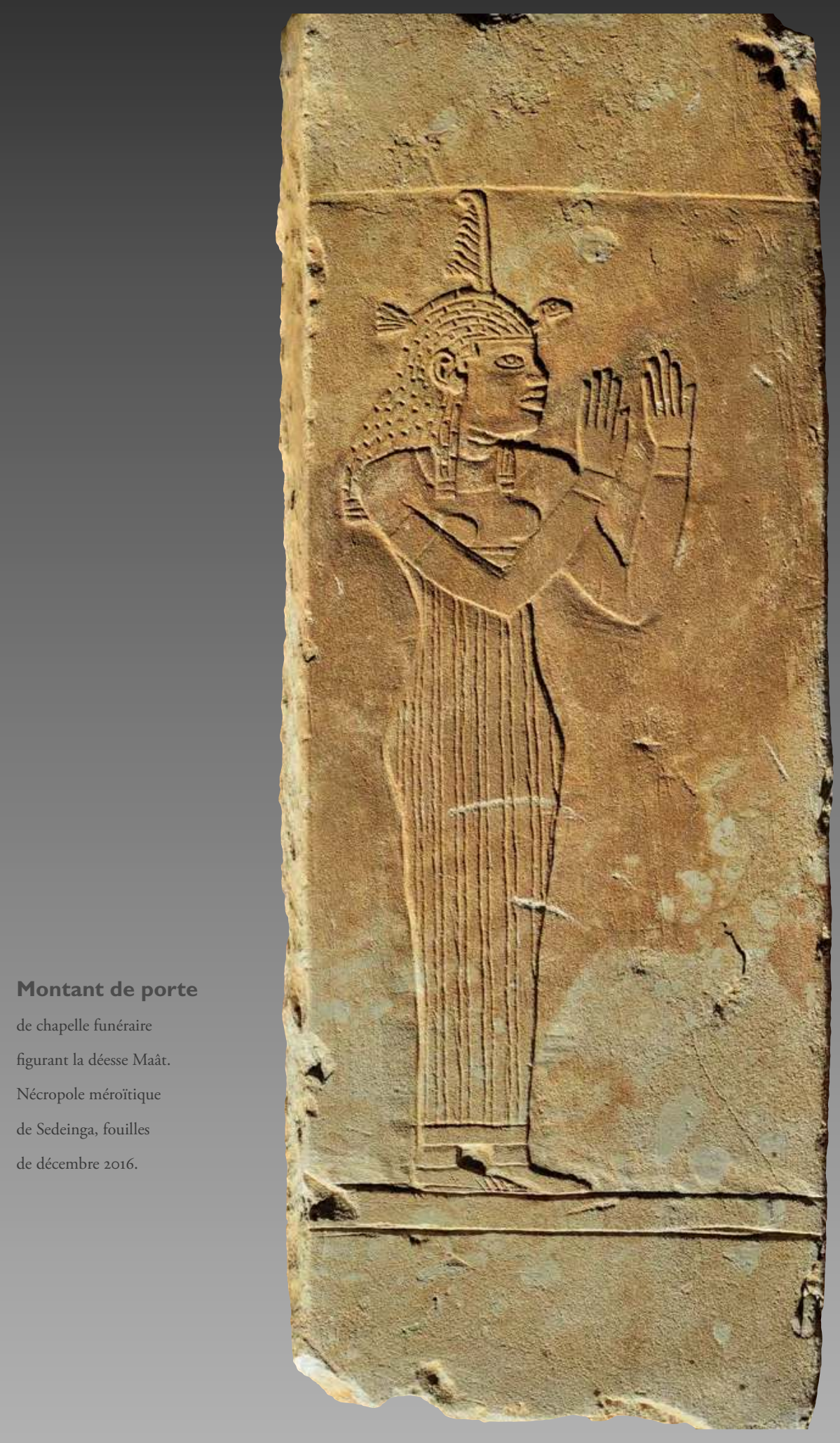




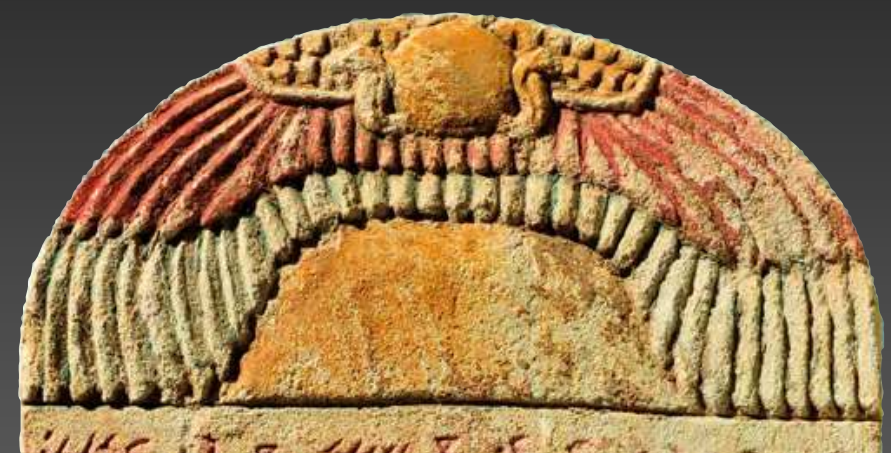

:4is iो 5 समा 5 क $313: 3,8$

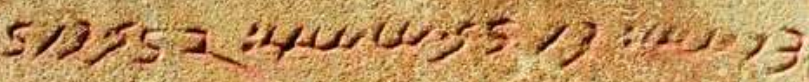

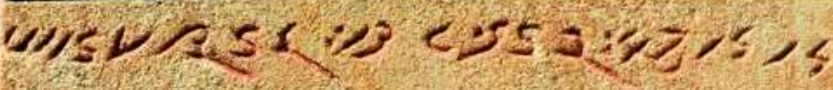

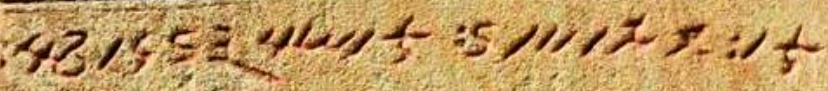

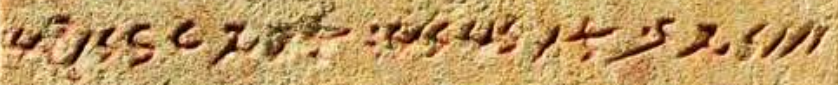

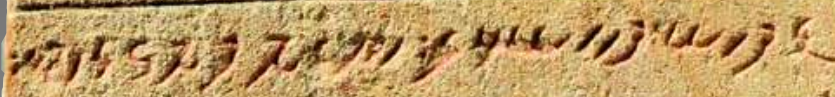

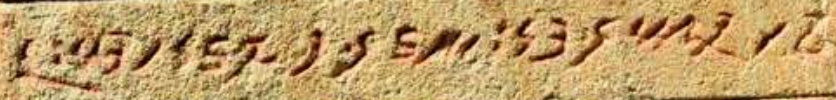

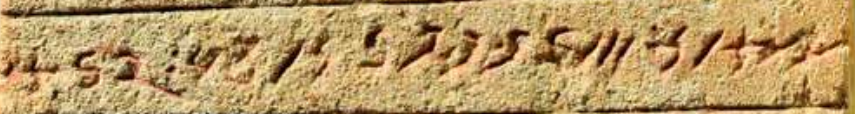

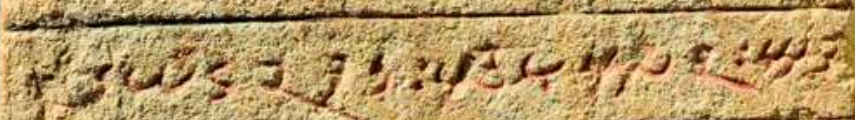

1. 2

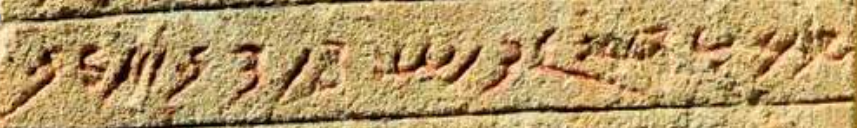

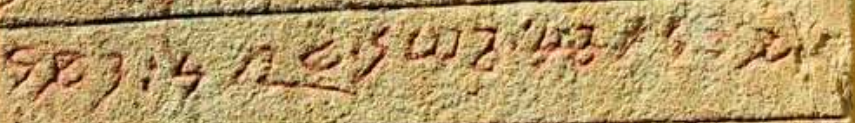

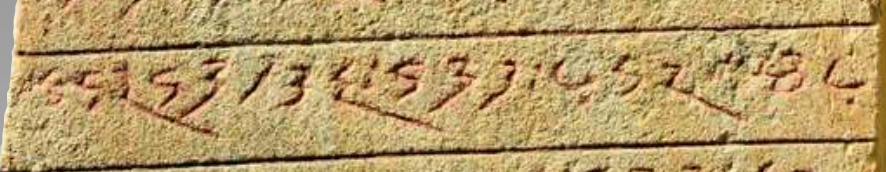

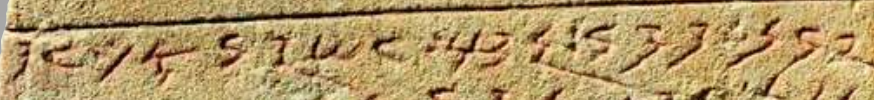

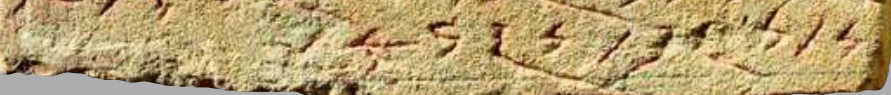

Stèle funéraire

de la dame Ataqéloula.

Nécropole méroïtique

de Sedeinga, fouilles

de décembre 2016 . 
Bien que Hintze eût banni les recherches sur la famille linguistique du méroïtique, affirmant longtemps que la langue était isolée en Afrique, puis, vers la fin de sa vie, que les conditions n'étaient pas réunies pour trancher en faveur de telle ou telle famille, les progrès trop lents de la traduction des textes incitèrent les chercheurs à mieux explorer cette piste. La filiation afro-asiatique (chamito-sémitique) chère à Zyhlarz avait été battue en brèche par Hintze dès 1955 et offrait peu de vraisemblance. En 1958, dans un court article intitulé "The Present State of Meroitic Studies», l'africaniste Werner Vycichl proposait de chercher du côté des langues subsahariennes.

Or, en 1963, le linguiste américain Joseph H. Greenberg fit paraître un ouvrage capital, Languages of Africa, qui regroupait toutes les langues d'Afrique en quatre superfamilles (ou phylums): l'afro-asiatique (que les francophones appellent souvent chamito-sémitique), le Niger-Congo (les langues apparentées au groupe bantou, depuis le Sénégal jusqu’à l'Afrique du Sud), le khoisan (langues "à clics» d'Afrique australe) et le nilo-saharien. Dans ce dernier phylum, Greenberg avait rassemblé un grand nombre de familles de langues d'Afrique orientale considérées auparavant comme indépendantes, notamment le nubien, le nilotique (dinka, massaï, etc.) et les langues sahariennes (toubou, kanouri, etc.). Le cœur du nilo-saharien était constitué d'un groupe appelé «soudanique oriental» (East Sudanic) où se retrouvaient le nubien, le nara (un parler isolé d'Érythrée), l'ensemble des langues nilotiques plus quelques groupes divers. Dès l'année qui suivit la parution de Greenberg, l'archéologue et méroïtisant Bruce G. Trigger publia dans la revue Kush un article important intitulé Meroitic and Eastern Sudanic: a Linguistic Relationship? Il y reprenait la comparaison du méroïtique et du nubien, y adjoignant plusieurs autres langues du groupe soudanique oriental comme le nara d'Érythrée. Malheureusement, nouveau venu dans l'arène des études nubiennes, il utilisa en partie, sans méfiance, les traductions erronées de Zyhlarz. C'en était trop pour Fritz Hintze, qui, lors de la première conférence d'études méroïtiques à Berlin en 1971, réfuta l'ensemble des conclusions de Trigger et se permit une parodie un peu cruelle, montrant qu'avec des méthodes aussi approximatives on pourrait relier le méroïtique aux langues altaïques (turc et mongol). Cette attaque en règle mit pour longtemps un terme aux recherches sérieuses sur la parenté du méroïtique.

La dernière décennie du $\mathrm{xx}^{\mathrm{e}}$ siècle vit l'étude du méroïtique tomber en léthargie. Fritz Hintze mourut en 1993 et Inge Hofmann arrêta ses publications la même année. Bruce G. Trigger s'était depuis longtemps tourné vers l'archéologie amérindienne. Nicholas B. Millet publia certes quelques inscriptions de Qasr Ibrim en 1991, mais de façon minimaliste. Le projet français du Répertoire d'épigraphie méroïtique (REM) était presque arrêté. Pourtant, c'est de France qu'allait venir un regain d'intérêt. En 1998, 
sous l'impulsion de Claude Carrier, le REM fut remis sur les rails et, finalement, les trois premiers volumes, fraîchement sortis des presses, furent offerts au professeur Leclant pour son quatre-vingtième anniversaire en juillet 2000. Dès 1999, j'inaugurai pour ma part une série d'articles consacrés à la langue et à l'écriture méroïtiques. En 2002, j'achevai un ouvrage, intitulé La langue du royaume de Méroé, paru en 2007, qui constituait la première synthèse d'envergure sur le méroïtique, son système graphique, sa grammaire et les méthodologies qui permettraient de progresser en ce domaine. La partie consacrée à la classification de cette langue dans une famille donnée n'était alors qu'une compilation de mes prédécesseurs, sans parti pris. Mais en 2004 fut menée une étude comparative qui confirmait, quarante ans plus tard, la thèse de Trigger: le méroïtique était bel et bien une langue nilo-saharienne, appartenant à la famille soudanique orientale et plus exactement à un groupe « soudanique oriental nord" (SoN) comprenant le nubien, le nara et deux autres groupes de langues parlées au Darfour et dans les Monts Nouba. Cette étude, enrichie des enquêtes linguistiques de terrain que j'ai réalisées en Érythrée et au Soudan, a débouché sur un volume publié en 2010, Le méroïtique et sa famille linguistique, qui ne présente plus des comparaisons isolées mais replace, selon les vœux de Hintze, le vocabulaire et la grammaire du méroïtique au sein des reconstructions que l'on peut établir pour l'ensemble du groupe Son.

Ce renouveau ne s'est pas cantonné à la France. L'Allemagne a repris la place qu'elle avait toujours tenue dans les études méroïtiques avec la publication, actuellement en trois volumes, par Jochen Hallof, des nombreux

des origines

à la chute

du sultanat

Fung textes inédits trouvés depuis 1976 dans les fouilles britanniques de l'Egypt Exploration Society à Qasr Ibrim, en Nubie égyptienne. Un ouvrage intitulé Einführung in die Meroitistik [Introduction aux études méroïtiques] a été publié en 2014 par Francis Breyer, reprenant, entre autres et avec quelques annotations critiques, mes travaux sur la langue de Méroé. On peut donc espérer que ce regain d'intérêt pour le méroïtique suscite de nouvelles vocations et permette des progrès accrus dans les années à venir.

\section{L'écriture méroïtique, une invention originale}

Quoique la langue méroïtique ait été présente dans la vallée du Nil moyen depuis au moins le deuxième millénaire avant notre ère, ce n'est qu'au III $^{\mathrm{e}}$ siècle av. J.-C. qu'elle fut dotée d'une écriture spécifique. Le royaume de Kerma, bien que constituant un Empire durable doté d'une administration centralisée, n'eut en effet recours à l'écriture que dans ses échanges diplomatiques avec les États du nord, notamment le royaume hyksôs de la XVI ${ }^{\mathrm{e}}$ dynastie, et ce certainement en recourant à des scribes égyptiens. L'utilisation 
de l'écriture avec parcimonie au Soudan ancien, que ce soit sous la XXV dynastie ou dans les royaumes de Méroé et de Napata, est peut-être un héritage de Kerma et l'oppose à l'Égypte, où le moindre site urbain livre des centaines de textes. Les fouilles récentes des villes méroïtiques de Mouweis par le Louvre ou de Hamadab par l'université Humboldt de Berlin n'ont, sur plusieurs années, mis au jour aucune trace d'écrit en dehors des temples.

On sait pourtant que l'éloquence était au Soudan ancien une qualité appréciée. En témoigne la stèle triomphale de Piankhy, certes composée par des scribes égyptiens, mais à l'initiative d'un monarque koushite. En témoignent également les graffiti tardifs du complexe d'Isis à Philae, rédigés en démotique par des scribes locaux mais sous la dictée de notables méroïtiques, et dont la volubilité contraste avec la sécheresse des inscriptions similaires laissées par les pèlerins égyptiens. Mais ce goût de l'éloquence allait sans doute de pair, comme souvent en Afrique subsaharienne, avec une grande importance de l'oralité et une certaine réticence devant la mise par écrit. Le nombre des textes méroïtiques illustre cette tendance: alors que le système d'écriture était d'une extrême simplicité et que certains graffiti maladroits montrent qu'il était connu en dehors des cercles lettrés, à peine plus de deux mille inscriptions ont été retrouvées en un siècle de fouilles. C'est bien peu pour six cents ans de civilisation méroïtique.

Linvention d'une écriture spécifique dans le royaume de Méroé peut être retracée dans ses grandes lignes, mais de nombreux détails, ici aussi, restent obscurs. Les Égyptiens ont introduit l'écriture au Soudan lors de leur colonisation du territoire sous la XVIII ${ }^{e}$ dynastie, mais uniquement pour les besoins de leur administration et la rédaction des textes sacrés dans les temples. Les élites acculturées pratiquaient l'égyptien et savaient l'écrire, souvent pour l'avoir appris dans le kap, cette école égyptienne pour les princes étrangers: dans la famille des Grands de Miam (actuel Aniba), Aménémopé, le futur «délégué de Koush", assistant du vice-roi Houy sous Toutânkhamon, commença sa carrière comme scribe auprès d'un vice-roi précédent. Sous la XXV dynastie puis le royaume de Napata, les textes sont nombreux mais toujours écrits en égyptien. Les rois font venir des scribes d'Égypte, qui enseignent leur art à des jeunes Koushites, ainsi que l'attestent les listes des hauts dignitaires des temples de Kawa et de Pnoubs (Doukki Gel) dans les stèles de l'an 3 d'Aspelta (vers 588 av. J.-C.), où des anthroponymes purement égyptiens voisinent avec des noms indigènes. Dans la seconde moitié de l'époque napatéenne, il est manifeste que les scribes sont presque entièrement de formation locale, comme en témoigne notamment la stèle de Nastasen, au milieu du Iv siècle avant notre ère, truffée de fautes d'égyptien dont les particularités trahissent la langue maternelle méroïtique du rédacteur. Lécriture est alors cantonnée aux établissements royaux et son seul vecteur est la langue égyptienne, ce qui oblige 
à traduire en langue sacrée une pensée exprimée en langue vulgaire, exactement comme on le fera dans l'Europe du Haut Moyen Âge, où l'expression écrite s'effectue exclusivement en latin. Sur des sites provinciaux éloignés du pouvoir central, il ne semble pas que cette pratique ait été connue. Ainsi, les grandes tombes napatéennes de la nécropole de Sedeinga, en Moyenne-Nubie, n’ont jusqu'à présent fourni aucune inscription en égyptien.

Toutefois, il était nécessaire dans ces textes napatéens de transcrire au moins quelques termes de la langue méroïtique ancienne: les noms de personnes, de tribus et de lieux. Pour cela, on recourait aux moyens que la tradition égyptienne mettait à la disposition des scribes. L'écriture syllabique, utilisée pour les noms étrangers particulièrement au Nouvel Empire, permettait en théorie de figurer les voyelles que l'égyptien de tradition, à l'instar de l'arabe et de l'hébreu, n'écrivait pas. Malheureusement, ses règles étaient fluctuantes et, surtout, mal connues à cette époque tardive. Aussi pouvait-on se contenter d'indiquer uniquement les consonnes. Mais dans la plupart des cas, les deux systèmes, syllabique et consonantique, sont mêlés inextricablement selon le bon vouloir du scribe. Il semble que pour les noms des souverains il y ait eu une doxa officielle, car les variations sont rares. En revanche, pour les particuliers, les graphies peuvent énormément varier d'un scribe à l'autre, ainsi que l'on peut le constater dans la transcription des noms des prêtres de la délégation royale dans les deux stèles de l'an 3 d'Aspelta, à Doukki Gel et à Kawa.

Il est à peu près certain que c'est de ce procédé de transcription des noms propres indigènes qu'est issu le système de l'écriture méroïtique, mais les étapes n'en sont pas encore claires, notamment parce que l'on n'a des origines à la chute du sultanat Fung pas retrouvé jusqu'à présent de traces de cursive égyptienne tardive sur le territoire de Koush. Seules des inscriptions hiéroglyphiques, gravées sur pierre ou sur métal, nous sont parvenues. Or, il est improbable que les Napatéens n'aient pas eu recours pour les textes courants au démotique qu'utilisent à cette époque les Égyptiens. On sait par exemple par les chroniques royales que les temples conservaient des archives qui étaient sans doute rédigées non en hiéroglyphes, écriture sacrée et de tracé complexe, mais en égyptien cursif, soit l'hiératique tardif ou le démotique ancien. Toutefois les fouilles archéologiques n'en ont pas livré jusqu'à présent, peut-être parce que le papyrus sur lequel elles étaient écrites se conserve difficilement au Soudan, où alternent fréquemment des chaleurs infernales et des pluies diluviennes. Il faut d'ailleurs supposer qu'il existait une forme de démotique particulière à Koush, dont les caractères sont à l'origine de l'écriture cursive méroïtique. On ne comprendrait pas sinon pour quelle raison, malgré un air de famille indéniable de la plupart des signes, certains autres présentent autant de divergences avec le démotique d'Égypte. 
L'écriture cursive méroïtique, contrairement à ce que l'on croyait il y a peu, est apparue avant son équivalent hiéroglyphique, selon un ordre inverse à ce qui s'était passé en Égypte trois millénaires plus tôt. Mais si en Égypte les écritures cursives (hiératique puis démotique) sont issues des hiéroglyphes par simplification graduelle des tracés, dans le royaume de Méroé les deux écritures sont nées indépendamment: la cursive est un développement local du démotique égyptien à visée utilitaire, tandis que l'hiéroglyphique est une transcription de la cursive à l'aide de signes empruntés au répertoire traditionnel des hiéroglyphes égyptiens. Cette dernière n'est d'ailleurs attestée que dans des contextes cultuels associés à la royauté. Comme nous l'avons vu dans le chapitre précédent (voir p. 208), c'est au règne d'Arnékhamani, vers 220 av. J.-C., que l'on doit désormais faire remonter le premier texte en cursive qui puisse être daté, mais il existe parmi les graffiti méroïtiques des temples de Kawa et de Doukki Gel des formes moins évoluées qui sont certainement plus anciennes: le nom d'Amon y est encore écrit au moyen d'un signe idéographique, alors que, sur le manche du sistre d'Arnékhamani, il est rédigé de manière phonétique, $(A) m n i$, comme il le sera systématiquement par la suite. Le dernier texte en cursive que l'on puisse dater serait l'inscription du roi blemmye Kharamadoyé, vers 410-420 de notre ère (voir chapitre suivant, p. 374 sq.), mais il est possible que certains graffiti de Philae soient plus tardifs de quelques décennies.

Le premier texte méroïtique hiéroglyphique connu est un double cartouche du roi Tanéyidamani, vers le milieu du $\mathrm{II}^{\mathrm{e}}$ siècle avant notre ère, gravé sur un cylindre de bronze provenant du Gébel Barkal. Les caractères sont encore hésitants et il faut attendre la fin du règne de ce souverain pour que le répertoire des signes hiéroglyphiques soit fixé, comme l'atteste une nouvelle version de son cartouche figurant sur sa grande stèle originaire du même site. Le cartouche de la reine Shanakdakhété dans le temple F de Naga, présenté jusque récemment comme le plus ancien texte méroïtique, doit être quant à lui replacé vers le début de notre ère: on se reportera à la section consacrée à cette souveraine dans le chapitre précédent (voir p. 261 sq.). Les textes écrits en hiéroglyphique sont peu nombreux et presque exclusivement constitués de légendes iconographiques des temples: même les chroniques royales sont rédigées en cursive. L'invention de cette écriture s'explique sans doute par la raréfaction des scribes capables d'écrire l'égyptien de tradition, utilisé pour les reliefs des temples jusqu’à la fin du II ${ }^{\mathrm{e}}$ siècle, par exemple dans le temple du Lion de Musawwarat. Elle permettait donc de faire figurer sur les parois des sanctuaires les signes sacrés égyptiens, revêtus d'un pouvoir magique, même s'il s'agissait d'une petite sélection et s'ils encodaient désormais, non la langue égyptienne, mais le méroïtique. Il semble d'ailleurs qu'il existait un tabou sur cette écriture, que le roman grec tardif d'Héliodore, 
les Éthiopiques, présente comme l'«écriture royale éthiopienne». Dans les rares contextes privés où elle est utilisée, notamment dans deux graffiti de Basse-Nubie, elle est mélangée à dessein avec des signes cursifs. Le dernier texte connu en écriture hiéroglyphique est une inscription sur un bol de bronze retrouvée dans la tombe d'un prince noba à el-Hobagi (voir p. 329, à la fin du chapitre précédent). Elle date du milieu du Iv ${ }^{e}$ siècle.

Comme dans toutes les écritures manuscrites, la forme des signes méroîtique a évolué depuis leur invention jusqu’à leur disparition. C'est surtout vrai de la cursive, l'écriture hiéroglyphique étant plus stable car fondée sur des représentations d'éléments empruntés au réel. Cette transformation progressive permet de dater avec une certaine précision les textes, à partir de tables paléographiques indexées sur les règnes connus, qui définissent trois grandes périodes : archaïque, transitionnelle et tardive, déjà stipulées par Griffith mais affinées depuis lors par la division de chacune en deux ou trois stades.

Un exemple particulièrement représentatif est celui du signe cursif transcrit $q$ (mais prononcé $/ \mathrm{k}^{\mathrm{w}} /$ ), composé de deux segments que l'on peut décrire (en n'oubliant pas que l'écriture se développe de droite à gauche) comme une courbe à laquelle est collé un triangle, suivie d'un trait vertical. Il provient d'une forme démotique locale issue du groupe hiéroglyphique égyptien $\square^{\natural}$ qui se transcrit $k \jmath$ mais se prononçait $/ \mathrm{ku} /$ en écriture syllabique. Dans les documents les plus anciens ( $\mathrm{III}^{\mathrm{e}}-\mathrm{II}^{\mathrm{e}}$ siècle av. J.-C.), le premier segment comprend un triangle énorme, et le trait, de petite dimension, est collé contre lui: \. À la fin de la période archaïque (vers 100 av.

des origines

à la chute

du sultanat

Fung J.-C.), le trait s'allonge et se détache et l'on trouve alors une forme $\$$ qui se maintient ainsi jusqu'au milieu du $\mathrm{I}^{\mathrm{er}}$ siècle de notre ère (période transitionnelle $\mathrm{B}$ ). Le triangle a ensuite tendance à s'affiner et à partir du $\mathrm{II}^{\mathrm{e}}$ siècle (période transitionnelle $\mathrm{C}$ ), il se transforme souvent en un simple trait $/ 7$. À partir du milieu du III ${ }^{\mathrm{e}}$ siècle (Tardif A), ce trait central devient largement majoritaire. Enfin, au début du Iv $\mathrm{V}^{\mathrm{e}}$ siècle (Tardif B), la courbe du premier segment se scinde en deux et le caractère est écrit /3. Il est donc possible, à partir de la forme de ce signe, mais aussi de plusieurs autres qui présentent semblablement une évolution régulière, de dater à cinquante ans près un texte méroïtique.

\section{Les principes de l'écriture méroïtique}

Les systèmes graphiques à travers le monde et les siècles se rangent en plusieurs catégories, que l'on peut classer grossièrement en deux groupes: les écritures logographiques, autrefois appelées idéographiques, qui encodent en priorité le sens des mots, chacun d'entre eux étant représenté par un signe 
spécifique, et les écritures phonétiques, qui encodent les sons de la langue. Parmi ces dernières, on distingue quatre grandes catégories: les syllabaires, les alphasyllabaires, les alphabets consonantiques (ou abjads) et les alphabets. Les syllabaires présentent théoriquement autant de signes qu'il y a de syllabes différentes dans la langue (généralement entre 40 et 60): on citera le linéaire B, écriture du grec mycénien, utilisé particulièrement en Crète, et les kanas japonais. Les abjads n'écrivent originellement que les consonnes et sont limités aux langues afro-asiatiques, notamment sémitiques, où les voyelles ne font pas partie des racines des mots: arabe, hébreu, araméen, etc. Les alphabets proprement dits écrivent de façon séparée les consonnes et les voyelles et sont tous issus, par leur généalogie ou leur principe, de l'alphabet grec.

Les alphasyllabaires utilisent un système à mi-chemin entre les syllabaires et les alphabets, d'où leur nom. Les signes de base correspondent à une syllabe dont la voyelle par défaut (ou voyelle inhérente) est presque toujours /a/: /ba/, /ta/, /sa/, etc. Si l'on veut écrire une syllabe dont la voyelle est différente, il faut introduire autour du signe de base un modificateur. Ainsi la syllabe /bi/ sera écrite /ba/ (signe de base + modificateur /i/). Ces modificateurs peuvent être placés au-dessus, en dessous, à droite ou à gauche des signes de base, chacun ayant une place spécifique (écritures indiennes), ou être inclus dans le signe (écritures éthiopiennes et vieux-perse).

Dans le cas du méroïtique, le modificateur suit simplement le signe de base comme dans un alphabet: il se place à gauche, puisque cette écriture était orientée de droite à gauche. Ainsi on écrit en cursive $\boldsymbol{\nu}$ (signe de base) pour /ba/ et $4 \mathcal{L}$ pour /bi/, avec adjonction du modificateur 4 /i/, et en hiéroglyphique 的/ba/ et 象放/bi/, le modificateur/i/ étant ici $\stackrel{\$}{\zeta}$. C'est cette succession linéaire générale des signes (elle concerne tous les modificateurs) qui avait conduit Griffith, le déchiffreur de l'écriture méroïtique, à penser qu'il s'agissait d'un alphabet, bien qu'il le jugeât "défectif», certaines voyelles "faibles» n'étant pas écrites selon lui. En fait, la voyelle non écrite est systématiquement /a/ et la nature de ce système a été tirée au clair par Fritz Hintze dans un article de 1973. Toutefois, le savant est-allemand n'alla pas jusqu'à proposer une réforme de la translittération que Griffith avait mise en place: chaque signe de base y est représenté en écriture romane par sa consonne. Ainsi $\}$, «dieu ", est translittéré conventionnellement $m k$ alors qu'il était écrit (et prononcé) ma-ka. Toutefois, comme nous le verrons, l'écriture méroïtique comporte des ambiguïtés qui empêchent dans bien des cas de rétablir une prononciation certaine. Aussi vaut-il mieux garder cette translittération conventionnelle qui a l'avantage de rendre exactement compte de la graphie des mots, signe par signe, même si elle ne correspond pas au système alphasyllabique de l'écriture. 
le Soudan

\section{0}

des origines

à la chute

du sultanat

Fung
Les alphasyllabaires sont généralement issus des abjads (alphabets consonantiques): les écritures indiennes, qui se comptent par dizaines, puisqu'elles comprennent la devanâgarî utilisée pour le sanscrit et l'hindi, mais aussi les systèmes tamoul, cinghalais, tibétain, birman, khmer, etc., sont toutes issues de l'écriture brahmi, qui s'est développée vers le $\mathrm{III}^{\mathrm{e}}$ siècle av. J.-C. à partir de l'écriture administrative de l'Empire perse, l'araméen, qui était un abjad. Le vieux-perse ou syllabaire persépolitain, bien que ses signes soient cunéiformes, est sans doute également un rejeton du système araméen, artificiellement élaboré pour doter la langue de Darius I ${ }^{\text {er }}$ d'une écriture propre. L'alphasyllabaire éthiopien dérive de l'écriture sudarabique, également un abjad. L'écriture méroïtique, quant à elle, provient principalement des caractères consonantiques simples qui constituent un abjad à l'intérieur du répertoire des signes égyptiens (aussi bien hiéroglyphiques que démotiques). Mais cet abjad ne constitue qu'une qu'une toute petite partie des caractères de l'écriture égyptienne, qui est un système mixte où voisinent des logogrammes, des signes exprimant plusieurs consonnes, d'autres une seule, et des compléments phonétiques destinés à lever des ambiguïtés. Lélaboration de l'écriture méroïtique a donc représenté un travail de simplification drastique à partir d'un système extraordinairement complexe constitué sur des millénaires.

Les alphasyllabaires comportent un grand avantage, puisque l'on économise l'écriture de tous les /a/ internes ou finals, mais leur découpage des mots en syllabes simples constituées d'une consonne et d'une voyelle entraîne quelques inconvénients. Il faut en effet recourir à des subterfuges pour transcrire une syllabe composée simplement d'une voyelle (notamment à l'initiale) ou des successions de consonnes. Chaque écriture a élaboré des procédés divers. En méroïtique, le même signe, $\$ 2$ en cursive, క్ำ en hiéroglyphique, était utilisé pour les /a/ et les /u/ (peut-être aussi /o/, dont l'existence n'est pas certaine) situés à l'initiale. Ainsi le nom du dieu Horus, prononcé /ara/ (du moyenégyptien $H r$ ) était-il écrit $\omega$ S2, en hiéroglyphique ॰ $\square$ \$़ी tandis que celui d'Osiris, prononcé /usuri/ (de l'égyptien $W s j r$ ), était-il écrit $4 \omega / 352$,

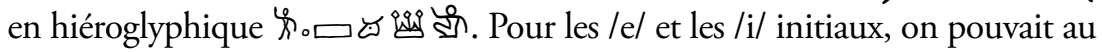
début utiliser les modificateurs $e$ et $i$ avec une valeur de signe de base, ce qui semble une entorse aux principes d'une écriture alphasyllabique, mais au fil des siècles on régularisa la situation en les faisant précéder d'un $y$, qui n'était pas prononcé mais servait de signe de base factice: ainsi $\$ 34 \mathrm{WS}$ erike,

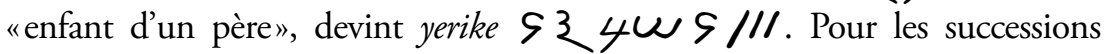
de consonnes, on introduisit entre elles le modificateur $e$, qui représentait la voyelle fermée /e/ du français «été» mais aussi la voyelle neutre /ə/ du français «revenir» et qui était ici muet, comme dans le français du Nord «bouleversant». Ainsi le nom de la cité de Qurta, en Basse-Nubie, connue en grec ancien sous la forme Kortê, est écrit en mérö̈tique $4 \cdot \zeta S \omega / 7$ Qoreti, où le signe $S e$ 
est un simple artifice pour noter la succession immédiate $\mathrm{du} / \mathrm{r} /$ et $\mathrm{du} / \mathrm{t} /$. Pour les nasales, $/ \mathrm{m} / \mathrm{ou} / \mathrm{n} /$, placées devant une consonne, on recourut à un procédé encore plus étonnnant mais attesté dans d'autres écritures comme le syllabaire chypriote, tout simplement en ne les écrivant pas: ainsi "Candace», "reine-

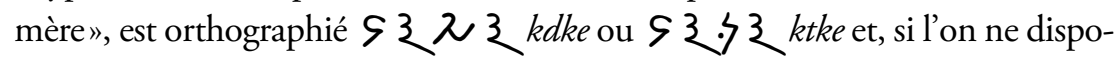
sait pas des transcriptions égyptienne kntjky, grecque kandakê et latine Candace, on ne saurait même pas que le mot comportait une nasale. Semblablement, les consonnes doubles ne sont pas répétées, mais écrites comme les consonnes simples: le groupe prononcé $/ \mathrm{k}^{\mathrm{w}} \mathrm{urra} /$, «le souverain», est ainsi orthographié $\omega / 7$ qor. Toutes ces conventions particulières, ainsi que plusieurs autres qu'il serait fastidieux d'énumérer ici, rendent difficile la reconstitution de la prononciation exacte du méroïtique.

Enfin, une originalité de cet alphasyllabaire est de posséder quatre signes à valeur vocalique fixe: on n'écrit jamais les syllabes $n e$, se, te et to en deux caractères $\left({ }^{*} \mathrm{n}+\mathrm{e},{ }^{*} \mathrm{~s}+\mathrm{e}\right.$, etc.) mais on utilise pour cela des signes spéciaux, respectivement $\beta, 3, \zeta, \leftarrow$ en cursive, $\approx$, 路, 河, $\square$ en hiéroglyphique. Cette particularité, qui rappelle les véritables syllabaires, s'explique sans doute par le fait qu'il s'agissait de suffixes ou de désinences verbales fréquentes.

\section{Liste des signes méroitiques}

Les valeurs phonétiques du tableau présenté sur la page suivante appellent quelques remarques. Tout d'abord, nous nous sommes contentés d'une notation phonologique (par convention entre traits obliques), sans rentrer dans le détail de la réalisation phonétique précise (qui serait entre crochets). Mais il n'est pas inutile de préciser deux d'entre elles. Le phonème mérö̈tique /s/ était prononcé [6], c'est-à-dire comme un «s» français partiellement chuinté. Cela explique que les Égyptiens aient hésité entre $s$ et $\breve{s}$ pour la transcription des mots koushites: le nom même de Koush est écrit Kus

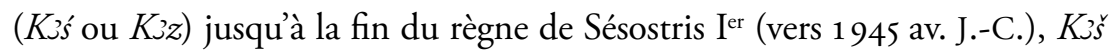
par la suite. D'autre part, le /d/ était réalisé entre voyelles comme une rétroflexe [d], c'est-à-dire avec le bout de la langue retourné contre le milieu du palais, ce qui produisait un son très proche d'un /r/ roulé. Par conséquent, les mots comprenant cette consonne sont écrits avec un /d/ en méroïtique mais avec un $/ \mathrm{r} /$ dans les transcriptions grecques et égyptiennes: la capitale est en méroïtique Medewi, en égyptien Mrw.t et en grec Méroê, d'où le nom français de «Méroé». Dans les deux cas, ces réalisations du /s/et du /d/ intervocaliques existent encore à l'identique dans plusieurs langues du Soudan, notamment le bedja et le nyimang. Il s'agit donc de traits aréaux (communs à une aire linguistique, même entre langues non apparentées). 
le Soudan

\section{2}

des origines

à la chute

du sultanat

Fung

\begin{tabular}{|c|c|c|c|}
\hline cursive & hiéroglyphique & translittération & valeur phonétique \\
\hline s2 & ज़ी & $a$ & /a/ ou /u/ initial \\
\hline$\nu$ & 市 & $b$ & /ba/ \\
\hline 2 & 庆 & $d$ & $/ \mathrm{da} /$ \\
\hline S & $\beta$ & $e$ & /e/, /ə/, ou absence de voyelle \\
\hline 3 & 25 & $b$ & $/ \mathrm{x}^{\mathrm{w}} /$ \\
\hline 4 & 歺 & $i$ & modificateur /i/ \\
\hline 之 & ३) & $k$ & $/ \mathrm{ka} /$ \\
\hline 3 & sa & $l$ & $/ \mathrm{la} /$ \\
\hline 3 & 承 & $m$ & $/ \mathrm{ma} /$ \\
\hline 及 & $\approx$ & $n$ & /na/ \\
\hline R & $\neq 7$ & ne & $/ \mathrm{ne} /, / \mathrm{n} /$ ou $/ \mathrm{n} /$ \\
\hline$/$ & 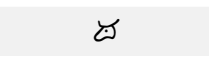 & $o$ & modificateur /u/ (peut-être aussi /o/) \\
\hline$<$ & $\oplus$ & $p$ & /pa/ (dans les emprunts égyptiens) ou /ba/ \\
\hline 17 & $\Delta$ & $q$ & $/ \mathrm{k}^{\mathrm{w}} \mathrm{a} /$ \\
\hline$\omega$ & $\circ \square$ & $r$ & $/ \mathrm{ra} /$ \\
\hline 3 & 迎 & $s$ & /sal \\
\hline VII & $\#$ & se & $/ \mathrm{se} /, / \mathrm{s} s /$ ou $/ \mathrm{s} /$ \\
\hline 4 & 3) & $t$ & $/ \mathrm{ta} /$ \\
\hline $1 \frac{\zeta}{5}$ & $\bar{\sigma}$ & te & $/ \mathrm{te} /, / \mathrm{ta} /$ ou $/ \mathrm{t} /$ \\
\hline$\longleftarrow$ & $\nabla$ & to & /tu/ (peut-être aussi /to/) \\
\hline 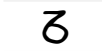 & 81 & $w$ & /wa/ \\
\hline$\subset$ & $\theta$ & $x$ & $/ \mathrm{xa} /$ \\
\hline$/ 1 /$ & 44 & $y$ & /ya/(?) \\
\hline : & $:$ & $:$ & séparateur de mots \\
\hline
\end{tabular}

Les valeurs des modificateurs vocaliques restent encore partiellement obscures, sans doute parce qu'elles ne reflètent qu'imparfaitement le système des voyelles méroïtiques. Il est évident, grâce aux transcriptions grecques et latines de noms propres et de toponymes, que le signe translittéré $e$ possède, comme en français, trois valeurs: (1) /e/ (entre "é» et «è»), (2)/ə/, le «e» atone de "revenir», (3) absence de voyelle, comme le «e muet» du français du Nord. Comme nous l'avons vu, cette dernière valeur permet d'écrire des consonnes nues, soit dans une suite de consonnes, soit en finale. En conséquence, les signes spécifiques ne, se et te ont aussi ces trois valeurs. Il est très probable, à la fois par la comparaison avec les idiomes apparentés et par déduction des quelques transcriptions grecques et latines dont nous disposons, que la langue méroïtique possédait les deux voyelles /o/ et $/ \mathrm{u} /$. Elles seraient dans la plupart des cas transcrites par le même signe ( $/$ en cursive, $\triangleleft$ en hiéroglyphique) que l'on translittère par convention $o$. 
Mais il est possible que certains /a/ internes aient été en fait des /o/: le nom du "pied", st, était prononcé plus probablement/sota/ que /sata/, comme le laisse supposer le proto-nubien *os-ti issu d'un ancien *so-ti.

Trois signes sont particulièrement attestés dans les emprunts à l'égyptien, $p, x$ et $h$. Il est quasiment certain que la langue méroïtique ne possédait pas de /p/, qui est absent des langues apparentées. Le signe translittéré $p$ devait donc être prononcé /b/ et faire double emploi avec le signe $b$, comme en témoignent certaines variations orthographiques (par exemple qorpse ou qorbse, "celui des rois»). Il est surtout utilisé à l'initiale, particulièrement lorsqu'il transcrit l'article égyptien $p_{3}$ dans les emprunts, mais aussi, par extension, dans des mots purement méroïtiques. Il est toutefois possible que les lettrés aient affecté une prononciation différente, à l'égyptienne, dans les emprunts tels que pelmos, «stratège», (de l'égyptien ṗ-mr-mš, "le général»).

Pour ce qui est des deux signes actuellement translittérés $x$ et $h$ (autrefois $\underline{h}$ et $h$ ), la situation n'est pas encore tout à fait claire et le court exposé suivant est le résumé d'une recherche récente et inédite. Il semble que, dans les emprunts égyptiens, ces deux signes étaient prononcés comme la fricative vélaire égyptienne $/ \mathrm{x} /$ (translittérée $h$ ), qui sonne comme le «ch» de l'allemand «Achtung» ou le «kh» de l'arabe «Khaled». Le second signe, $h$, se trouve plutôt devant les voyelles arrondies comme /u/ et /o/: ainsi le nom de personne Phome, «Pacôme», (de l'égyptien Pз- 'blm "faucon sacré») ou le toponyme Phrse, «Pakhôras", (transcription grecque), actuel Faras. Mais dans les mots purement méroïtiques, ces deux caractères servaient sans doute à transcrire la nasale vélaire $/ \mathrm{y} /$, c'est-à-dire le "ng" de l'anglais «king»: ainsi le verbe "boire», he, devait se prononçait $/ \mathrm{ge} /$ ou $/ \mathrm{g}^{\mathrm{w} e} /$, à comparer avec le vieux-nubien $\boldsymbol{c} \mathbf{\boldsymbol { l }}$ - prononcé /gi/. C’est d'ailleurs du signe méroïtique $\subset x$ que dérive la lettre $\boldsymbol{\Gamma}$ qu'utilisait le vieux-nubien au Moyen Âge pour écrire la nasale vélaire / $\mathrm{y} /$. Dans le nom de la reine Amanishakhéto "Amon l'a engendrée», Amni-sxe-to, le radical verbal écrit sxe- était sans doute prononcé/say/ou /soy/et serait en ce cas apparenté au nubien du Kordofan šiz-, "accoucher», ou au nyimang suyo, "enfanter, engendrer». Les transcriptions égyptiennes d'époque méroïtique utilisent d'ailleurs le digramme $n \underline{h}$ ou $n \underline{b}$ pour cette nasale vélaire: Jrnh-Jmn pour le nom du roi Arnékhamani, méroïtique Elxmni, Jrknhbrr pour le prince Arikankharor, méroïtique Arikxror. La différence entre $x$ et $h$ en ce cas n'est pas encore bien éclaircie. Qu'un caractère puisse avoir dans une langue deux lectures phonétiques suivant son étymologie est bien attesté ailleurs, notamment en français, où le digramme "ch" est prononcé /š/ dans les mots du fonds latin, mais $/ \mathrm{k} /$ dans les termes empruntés au grec (archéologie, orchestre, etc.). 
Enfin, il n'est pas sûr que le caractère $y$ ait eu la même valeur que son étymon égyptien, c'est-à-dire la semi-voyelle /y/ (notée [j] en alphabet phonétique international). Nous avons vu précédemment qu'elle pouvait servir de signe de base factice pour les voyelles initiales. Elle a aussi ce rôle à l'intérieur des mots: Asoreyi, «ô Osiris!», était prononcé /usurii/. De plus, cette semi-voyelle est généralement absente des langues apparentées. On peut donc légitimement se demander si le caractère $y$ avait une valeur phonologique en méroïtique ou s'il n'était pas plutôt un instrument commode pour pallier les inconvénients inhérents au système alphasyllabique.

\section{Liste des signes numéraux méroïtiques}

le Soudan

\section{4}

des origines

à la chute

du sultanat

Fung

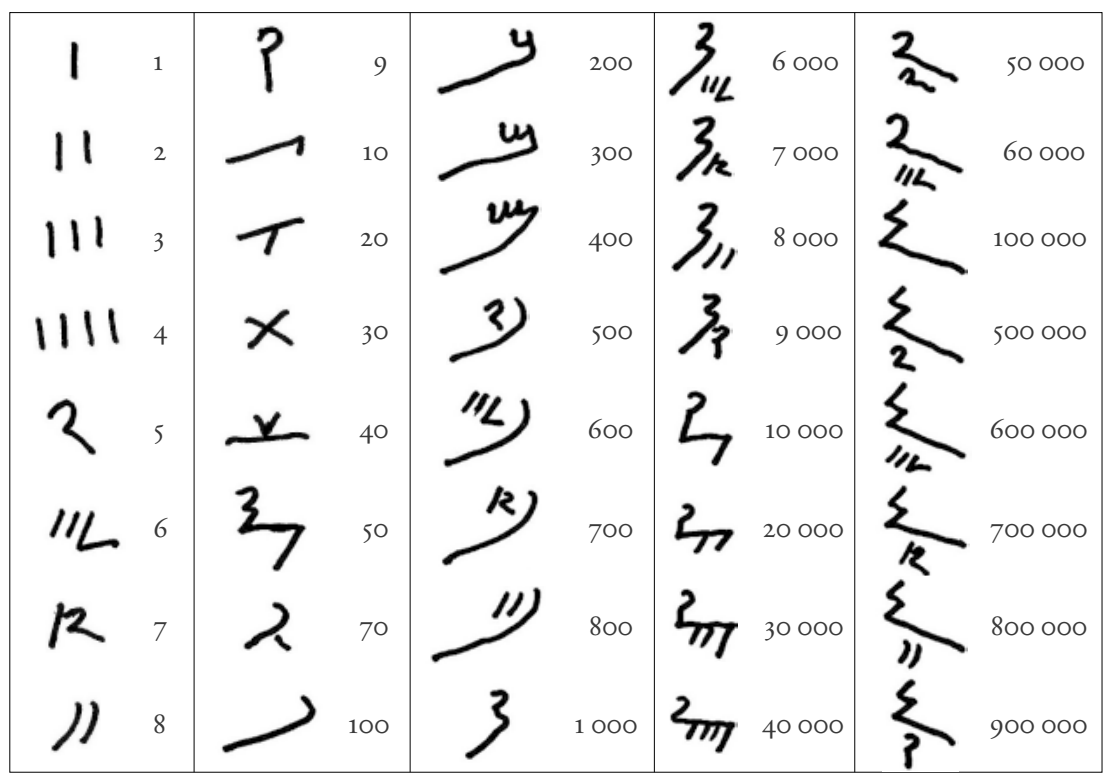

Les chiffres ne sont pas tous connus: on ignore par exemple ceux pour 60, 80 et 90 . Griffith en avait établi une liste dont il avait déduit les valeurs à partir des signes égyptiens (démotiques et hiératiques). Tout récemment, parmi la documentation de Qasr Ibrim, Jochen Hallof a publié un ostracon (tesson inscrit) qui semble un exercice scolaire de numération ou un pensebête et qui livre une suite de chiffres depuis les fractions jusqu'à 900000 , à raison de cinq signes par rang de chaque classe. Le nombre des chiffres connus a donc fortement augmenté, mais on ignore toujours les signes pour 60,80 et 90. En revanche, les unités manquantes de centaines, milliers, dizaines et centaines de milliers peuvent être facilement inférées à partir de celles que l'on connaît puisqu'elles incluent les signes des unités simples. Mais, surtout, trois signes ont pu être rectifiés: le 5 de Griffith était 
en fait un 7, son 8 était un 5 et son 7 était un 8. Par contre, il n'y a pas eu de rectification pour les fractions, qui sont marquées par des séries de points dont chacun représente $1 / 12^{\mathrm{e}}$.

\section{La langue méroitique : quelques éléments de grammaire}

Il n'est évidemment pas possible dans le cadre de ce chapitre de donner un aperçu complet de la langue de Méroé: on se référera à nos ouvrages de 2007 (La langue du royaume de Méroé) et de 2010 (Le méroïtique et sa famille linguistique) pour un examen détaillé. On se contentera ici de quelques remarques générales et de quelques exemples choisis.

Le méroïtique est une langue de type agglutinant, comme le sumérien, le turc, le hongrois, le basque ou la plupart des parlers bantous, bien qu'il n'y ait pas entre elles de lien génétique. Dans ces langues, les différents traits grammaticaux sont indiqués par des suffixes généralement invariables qui s'ajoutent en chaîne après le radical du nom ou du verbe. Toutefois, en méroïtique, de fréquents phénomènes d'assimilation des consonnes brouillent les frontières entre ces suffixes. L'ordre des mots est assez rigide: le sujet d'une phrase est en tête et le verbe à la fin, le déterminant (article, adjectif) est placé après le déterminé (nom), on trouve des postpositions au lieu de prépositions. Les préfixes (par exemple la négation $m$ - formant des antonymes) sont l'exception, alors que les suffixes sont la règle.

On connaît actuellement en méroïtique des substantifs, quelques adjectifs, des articles, des verbes, des postpositions, une conjonction de coordination (kelw "et aussi»). Les seuls pronoms identifiés de manière sûre sont les démonstratifs qo, "celui-ci, celle-ci», pluriel qoleb. Le seul adverbe connu avec certitude est $d i k$, «sur toute l'étendue» pour préciser un parcours entre deux lieux. Aucune conjonction de subordination n'a été jusqu’à présent repérée.

Le substantif est formé sur des racines de type divers comprenant deux consonnes radicales et une ou deux voyelles. Contrairement à l'égyptien, ces voyelles appartiennent à la racine: ainsi, $k d-i$ prononcé $/ \mathrm{kadi} /$, "femme», et ked-/ked/, " massacrer", sont différenciés par leur voyelle radicale. À l'exception des mots d'origine étrangère ( $n b r$ /nabara/, "or», emprunté à l'égyptien $n b w$, tlt /talanta/, "talent», emprunté au grec talanta), les radicaux qui comprennent plus de deux consonnes sont théoriquement suffixés ou composés: kdise/kadis/, " sœur», est ainsi issu de kdi, «femme».

L'article est $-l$ (prononcé $/ \mathrm{la} /$ ) ou -li (sans doute /lai/). Il n'a pas vraiment de valeur sémantique (indéfini et défini, comme en français) mais constitue plutôt un outil syntaxique. Il est placé à la fin du groupe nominal, quelle qu'en soit la longueur: $k d i-l$, «la femme» ou " une femme», $k d i-l h-l$, 
le Soudan

\section{6}

des origines

à la chute

du sultanat

Fung «la grande femme», $k d i-s e-l$, "chaque femme»; ant-li, "le prêtre» ou «un prêtre», ant Mni-se-l, "le prêtre d'Amon». Si le substantif est terminé par une consonne, il y a souvent assimilation, régressive ou progressive: qore $/ \mathrm{k}^{\mathrm{w}} \mathrm{ur} /$, " souverain ", qor /kwurra/, " le souverain» $(/ \mathrm{r} /+/ \mathrm{l} />/ \mathrm{rr} /$ par assimilation progressive), wide /wid/, "frère", wil/willa/, " le frère " $(/ \mathrm{d} /+/ 1 />/ 11 /$ par assimilation régressive). Si cette consonne finale est un $/ \mathrm{s} /$, la séquence $/ s /+/ 1 /$ devient $/ \mathrm{t} /$ à partir du i ${ }^{\text {er }}$ siècle de notre ère (loi de Griffith) : kdise /kadis/, " sœur», kdit/kadita/, «la sœur» (originellement/kadisla/). Le pluriel des substantifs est le plus souvent marqué par l'article au pluriel -leb /leba/, «les» ou «des»: kdi-leb, «les femmes» ou «des femmes».

Le génitif (complément de nom) existe sous deux formes: un génitif direct, antéposé, sans marqueur spécifique, mais où l'article suit généralement le nom déterminant: pelmos-li sem-l, «l'épouse d'un stratège » (lit. "stratège-un épouse-la»), et un génitif indirect, postposé, utilisant la postposition -se, équivalant au français «de»: ant Wos-se-l, "un prêtre d'Isis" (lit. "prêtre Isis-de un».

Les phrases nominales, très fréquentes dans les textes funéraires, utilisent une copule -o ou -owi au singulier: "c'est», -kwi au pluriel: «ce sont». Le groupe nominal ainsi prédiqué est obligatoirement suivi de l'article. On trouve ainsi pesto-l-o, "c'est un vice-roi», apote-leb yetmde-leb$k w i$, "ce sont des neveux d'ambassadeurs" (lit. "ambassadeur-des neveudes ce sont»). Si c'est un nom propre qui est ainsi prédiqué, on utilisera qo, sans doute issu de qo+-o, "celui-ci/celle-ci est»: Amnisxeto qo, "celle-ci est Amanishakhéto", "c'est Amanishakhéto".

Le méroïtique utilise non des prépositions comme le français ou l'anglais, mais des postpositions, placées après le nom. Outre la postposition génitivale -se, "de», mentionnée ci-dessus, on trouve -te, "dans", marque du locatif, ex. : Wos Pilqe-te "Isis de Philae», lit. "Isis dans Philae»; -w «à», "pour», ex.: $m k-l-w$, «pour le dieu»; -ke, «depuis» (anglais "from») ex.: Medewi-ke, "depuis Méroé»; -yte, "jusqu’à», ex. : Selele-yte "jusqu’à Shellâl», et plusieurs postpositions composées: $-n-l-w$, «en présence de», -se-l-w, «sous l'autorité de».

La morphologie verbale reste actuellement très peu connue, parce qu'elle n'est guère présente que dans les textes royaux, qui sont les moins accessibles des inscriptions méroïtiques. Seuls quelques verbes sont traduits: (e)l-, «donner», ked-, "massacrer» (sans doute aussi "découper»), are-, "prendre», tk(e)-, "aimer». Dans les narrations, les formes verbales comportent des préfixes qu'il faut sans doute interpréter comme d'anciens pronoms que les scripteurs n'ont pas disjoints des verbes: ainsi, dans la grande stèle de Hamadab, abr-se-l ye-ked-i signifie sans doute "j'ai massacré chaque homme» (lit. "homme-chaque-un je-massacrai»), 
où ye devait être originellement être le pronom sujet de la $1^{\mathrm{e}}$ personne. Il semble que l'impératif soit marqué par une désinence -te au singulier, au pluriel -k(e)te: ex. pwrite $l-x$-te, "donne-lui la vie» (lit. "vie donnez-à lui»), ato mhe pso-he-kete, "faites-lui boire de l'eau abondante» (lit. "eau abondante faites-boire»). La recherche sur la morphologie verbale est un vaste champ inexploré qui sera sans doute la prochaine étape dans l'étude du méroïtique, grâce notamment à la comparaison avec les langues dont l'apparentement a été récemment mis en évidence.

\section{Petit glossaire méroïtique}

La liste suivante inclut la plupart des termes méroïtiques pour lesquels la traduction présente un haut degré de vraisemblance. Ils sont donnés ici sous leur graphie la plus fréquente mais nombre d'entre eux présentent des variantes, comme kdwe et ktwe pour $k d k e$, "Candace», qui ne sont pas systématiquement précisées. La transcription phonétique entre crochets est encore souvent incertaine, pour des raisons que nous avons énoncées dans la section consacrée à l'écriture. Par précaution, nous avons conservé aux signes $x$ et $h$ les valeurs $[\mathrm{x}]$ et $\left[\mathrm{x}^{\mathrm{w}}\right]$ qui sont bien attestées dans les emprunts à l'égyptien, la consonne $[\mathrm{x}]$ étant le « $\mathrm{kh}$ » de l'arabe Khaled, bien qu'il semble que, dans certains cas, il s'agissait d'une nasale vélaire («ng» de l'anglais king): cette nouvelle interprétation, exposée dans l'introduction de notre tableau des signes ci-dessus, est en effet un travail en cours. La voyelle [ə] est un "e" atone comme dans le français «le»; [6] est un "s» légèrement chuinté; [d] est un «d» rétroflexe, produit avec la langue retournée contre le palais, acoustiquement proche d'un «r» roulé.

\begin{tabular}{|c|c|c|}
\hline abese & [əbece] & gazelle \\
\hline abore & [ambur] & éléphant \\
\hline$a b r$ & [əbara] & homme (mâle) \\
\hline ant & [annata] & prêtre (< égyptien) \\
\hline apede & [əbede] & Créateur \\
\hline apote & [upute] & ambassadeur (< égyptien) \\
\hline$a r$ & [ara] & jeune garçon \\
\hline are- & {$[\mathrm{ar}]$} & prendre, recevoir \\
\hline ark- & [arak] & piller, razzier \\
\hline asr & [ว6ara] & viande, animal \\
\hline$a t$ & [utta] & pain \\
\hline ato & {$[\mathrm{attu}]$} & eau \\
\hline bohe- & {$\left[\mathrm{bux}^{\mathrm{w} e}\right]$} & régner \\
\hline dime & {$[\operatorname{dim}]$} & vache \\
\hline
\end{tabular}


le Soudan

\section{8}

des origines

à la chute

du sultanat

Fung

\begin{tabular}{|c|c|}
\hline dmkte & [damakat] \\
\hline$d x e-$ & [daxe] \\
\hline erike- & [erikə] \\
\hline he & {$\left[\mathrm{x}^{\mathrm{w}} \mathrm{e}\right]$} \\
\hline$h r$ & [xwara $]$ \\
\hline$k d i$ & [kadi] \\
\hline kdite & [kadit], kdise [kadic] \\
\hline kdke, ktke & [kandake] \\
\hline ked- & [keda] \\
\hline kelw & [kelawa] \\
\hline$k l$ & [kala] \\
\hline (e)l- & [(e)la] \\
\hline lh & {$\left[\operatorname{lax}^{\mathrm{w}} \mathrm{a}\right]$} \\
\hline$m d x e$ & [madaxe] \\
\hline mete & [məte] \\
\hline mhe & {$[\operatorname{maxu}]$} \\
\hline$m k$ & [maka] \\
\hline mlo & [malu] \\
\hline mlowi & [malui] \\
\hline$m s$ & [maca] \\
\hline mte & [mate], mse [mace] \\
\hline$n b r$ & [nabara] \\
\hline nob & [nuba] \\
\hline nse & [nac] \\
\hline ntke & [natake] \\
\hline pelmos & [balamuca] \\
\hline
\end{tabular}

perite [bərit]

peseto [bacentu], pesto [bacantu]

pete [bette]

pi [bi]

pqr [bakwara]

pwrite [bawarit]

qebese [kwəbə6]

qelile [kwolil]

qese [kwa6]

qo $\quad\left[\mathrm{k}^{\mathrm{w}} \mathrm{u}\right]$

qore $\quad\left[\mathrm{k}^{\mathrm{w}} \mathrm{ur}\right]$

qoresem [kwurə6ema] offrande

enfanter

engendrer

boire

nord

femme

sœur

Candace, reine-mère

massacrer, découper

et aussi

nourriture

donner

grand, vieux

vierge (adj.)

jeune $(<m t e)$

abondant, beaucoup

dieu, déesse

bon, beau

santé

soleil

enfant

or (< égyptien)

esclave, d'où Noba

(locuteur de langue nubienne)

sacrifice

force

stratège, chef de nome

(< égyptien)

administrateur de temple

(< égyptien)

vice-roi (< égyptien)

serpent

lieu, endroit

prince

vie, force vitale

leur (adj. possessif)

collier

son (adj. possessif)

ce, cette; celui-ci, celle-ci

souverain (roi ou reine)

épouse royale; prince consort 


\begin{tabular}{|c|c|}
\hline$s d k$ & [6adaka] \\
\hline se & [6e] \\
\hline sem & [6ema] \\
\hline ssor & [6a6ura] \\
\hline$s t$ & [6ata] \\
\hline ste & [6ate] ou [6ote] \\
\hline$t d x e$ & [tadaxe] \\
\hline teneke & [tenekə] \\
\hline telepi & [teləbi] \\
\hline tewiseti & [twi6ti] \\
\hline tke- & {$[\mathrm{tak}]$} \\
\hline$t k k-$ & [takaka] \\
\hline tlt & [talanta] \\
\hline tmey & [tamia] \\
\hline tre- & {$[\operatorname{tar}]$} \\
\hline wte & {$[$ wat $(ə)]$} \\
\hline wide & {$[\operatorname{wid}(ə)]$} \\
\hline wle & [wal] \\
\hline$x l b i$ & [xalabi] \\
\hline$x r-$ & [xara] \\
\hline xrpxne & [xarabaxan] \\
\hline yed & [eda] \\
\hline yer & [era] \\
\hline yetmde & [etamace] \\
\hline yireqe & [irku] \\
\hline yirewke & [irəwak] \\
\hline
\end{tabular}

voyage

chaque

épouse

scribe (< égyptien)

pied, paire de pieds

tuteur, mère

enfant (d'une mère)

ouest

grenier, conteneur à grain

adoration (< égyptien)

aimer

piller, razzier

talent, unité de poids (< grec)

homme blanc (< égyptien?)

donner, offrir

vie, existence

frère

chien

taureau

manger (du dur)

gouverneur (< égyptien)

argent (métal ; < égyptien)

lait

neveu, nièce (individu plus jeune

dans la lignée maternelle)

hiéroglyphes

sud

est (point cardinal)

noms de divinités

$\begin{array}{ll}\text { Amésémi } & \text { [əmecemi] } \\ \text { Amnbse } & \text { [amannaba6] } \\ \text { Amni } & \text { [amanai] } \\ \text { Amnote } & \text { [amannute] } \\ \text { Amnp } & \text { [amanaba] } \\ & \\ \text { Amnpte } & \text { [amannabatte] } \\ \text { Apedemk } & \text { [əbedemaka] } \\ \text { Aqedise } & \text { [akwodic] } \\ \text { Ar } & \text { [ara] }\end{array}$

Amésémi (parèdre d'Apedemak)

Amon de Pnoubs (< égyptien)

Amon (< égyptien)

Amon de Thèbes (< égyptien)

Amon de Louqsor (confondu

avec le suivant) (< égyptien)

Amon de Napata (< égyptien)

Apédémak

Khonsou, également forme

lunaire d'Amon

Horus (< égyptien) 


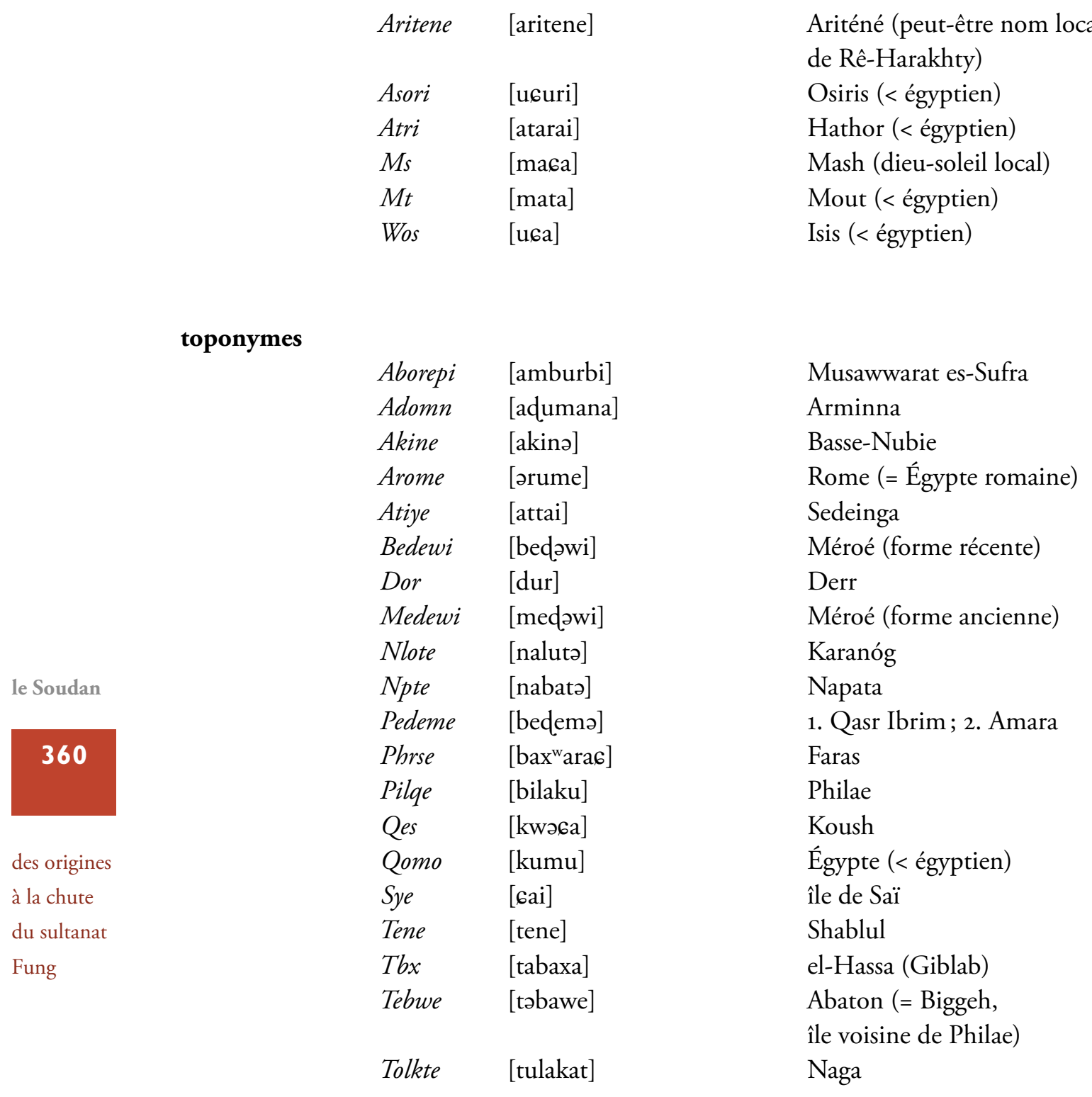




\section{À quelle famille de langues appartient le méroïtique?}

Ainsi que nous l'avons relaté dans nos sections précédentes sur le déchiffrement du méroïtique et la recherche ultérieure, la question des langues apparentées a joué un rôle central, bien souvent plus dommageable qu'utile, dans le long chemin vers la traduction des textes. Mais, étant donné la disparition sans descendance de l'idiome de Méroé et l'absence de textes bilingues, il était inévitable que les savants cherchent des informations en dehors de l'analyse interne des textes, qui avait démontré ses limites. D’autres langues anciennes avaient été déchiffrées en grande partie par la méthode comparative. L'akkadien par exemple avait bénéficié de la comparaison avec les autres langues sémitiques. Le hittite était resté longtemps une énigme, jusqu’à ce que le philologue autrichien Bedřich Hrozný s'avisât en 1916 de le comparer avec les langues indo-européennes, malgré le scepticisme de ses collègues, ce qui guida les premiers pas vers le déchiffrement complet de la langue.

On se souvient que Griffith avait dans un premier temps privilégié la piste nubienne. Les langues nubiennes, parlées en Égypte et au Soudan, présentent en effet de nombreux traits communs avec le méroïtique. Mais l'examen du vocabulaire s'était révélé décevant. Griffith était notamment frappé de ce que le méroïtique possédât deux racines pour «engendrer» (erike-) et "enfanter» $(d x e-)$ qui ne ressemblaient guère aux et de la mère), en nobiin et en vieux-nubien unn- et en dongolawi uski. Il ne pouvait savoir que les dialectes nubiens du Kordofan, alors presque inconnus, possédaient un verbe irlirki signifiant "enfanter", "engendrer» qui expliquaient le vieux-nubien ir-kane "naissance», présent dans le Lectionnaire (recueil de textes pour les lectures des offices) qu'il avait pourtant publié en 1913. Sinon, le lien avec le méroïtique erike- lui aurait sauté aux yeux.

Aussi, après lui, privilégia-t-on la piste couchitique, en cherchant notamment du côté du bedja, du somali, de l'afar, etc. et ce malgré le peu de similitudes que présentent dans leurs structures ces langues avec le méroïtique. Fritz Hintze par la suite démontra que cette piste était illusoire, mais ferma la porte à toute comparaison en décrétant que la langue de Méroé était un isolat linguistique, comme le sumérien ou l'étrusque, auxquels on ne connaît aucune langue apparentée. Il réfuta notamment la tentative de la relier au groupe «soudanique oriental» (l'Eastern Sudanic de Greenberg), rameau central des langues nilo-sahariennes, que développa Trigger en 1964 dans un article, il est vrai mal étayé. Plus tard, dans une étude de 1989, Hintze revint sur ce problème et, sans rejeter totalement 
l'hypothèse, précisa que l'on ne pouvait la prouver qu'en comparant le méroïtique à des formes reconstruites du soudanique oriental, c'est-à-dire en restituant tout d'abord les mots de la langue primitive à partir des langues actuelles, comme on l'a fait depuis longtemps pour l'indo-européen.

C'est cette méthode qui m'a permis de confirmer dans mon ouvrage, publié en 2010, Le méroïtique et sa famille linguistique - et de manière probante cette fois —, la thèse initialement soutenue par Bruce Trigger. Mes recherches ont bénéficié d'une situation beaucoup plus facile qu'en 1964. D'une part, le méroïtique, sa morphologie et son vocabulaire sont mieux connus. D'autre part, les efforts des linguistes de terrain pour étudier les langues nilo-sahariennes, dont les locuteurs sont souvent isolés et en petit effectif, ont permis d'en publier des descriptions plus précises et plus nombreuses que celles dont on disposait dans les années 1960.

Le nilo-saharien, le dernier des phylums (ou superfamilles, voir p. 343) postulés par Greenberg dans sa classification des langues d'Afrique, compte environ 120 idiomes très divers, répartis du nord au sud entre l'Égypte et la Tanzanie, de l'ouest à l'est entre le Niger et l'Éthiopie. Il se subdivise en plusieurs grandes familles (saharien, soudanique central, soudanique oriental), quelques groupes moins conséquents (maba, koman, kado) et plusieurs langues isolées (four, berta, kunama, gumuz). La famille soudanique orientale est le cœur du nilo-saharien et compte à son tour plusieurs groupes: le soudanique oriental nord, le nilotique, le surmique et des langues ou groupes dialectaux isolés: le jebel, le temein, le dadjo (ou daju). Le nilotique est un vaste ensemble réparti sur plusieurs pays, dont les

des origines

à la chute

du sultanat

Fung langues les plus connues sont le massaï (Kenya/Tanzanie), le dinka (Soudan du Sud), le luo (Kenya, ethnie du père de Barack Obama). Le surmique est également représenté par de nombreuses langues parlées au Soudan du Sud et en Éthiopie, dans la vallée de l'Omo. Le groupe surmique le plus connu est la tribu des Mursi, célèbres pour les plateaux insérés dans la lèvre inférieure que portent les femmes.

Le soudanique oriental nord (en abrégé Son) est constitué de cinq langues ou groupes de langues: le méroïtique, le nara, le nubien, le taman et le nyima, qui s'articulent généalogiquement comme présenté page ci-contre.

— Le nara, autrefois appelé "barya», est parlé en Érythrée par environ 50000 personnes au nord de la ville de Barentu, non loin de la frontière avec le Soudan.

- Le groupe nubien, parlé par 900000 locuteurs, présente une variété importante, que l'on pourrait comparer au groupe des langues germaniques. Il compte une langue ancienne, le vieux-nubien, écrite à l'aide de caractères coptes complétés de quelques signes méroïtiques. Elle était parlée 


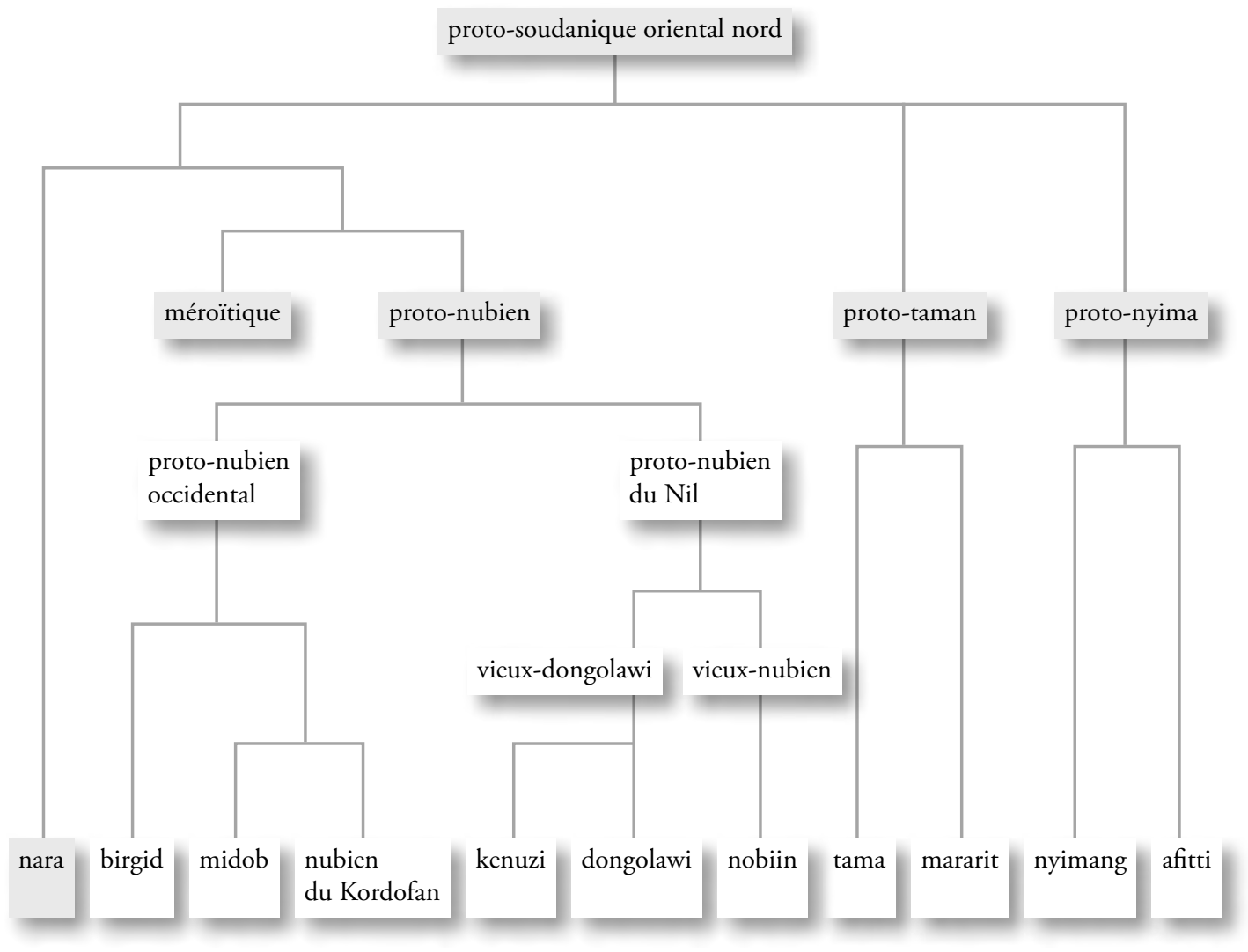

au nord du royaume chrétien de Makouria, entre la première et la troisième cataracte du Nil. La littérature connue est pour l'essentiel constituée de textes religieux. Son descendant actuel est le nobiin, parlé sur le même territoire et souvent appelé "mahasi» au Soudan. Entre la troisième cataracte et la grande boucle du fleuve, de part et d'autre de la ville de Dongola au Soudan, se situe le dongolawi (appelé localement «andaandi»), une langue nubienne assez différente: il n'y a pas d'intercompréhension avec les locuteurs du nobiin. Une ancienne colonie venue de Dongola au $\mathrm{XIV}^{\mathrm{e}}$ siècle de notre ère s'est établie près d'Assouan en Égypte, introduisant la langue dongolawi qui a évolué en un dialecte légèrement différent, le kenuzi (appelé localement «mattoki»). Les autres langues nubiennes sont parlées à l'ouest du Soudan: au nord des Monts Nouba, dans la province du Sud-Kordofan, se situe un ensemble de dialectes désigné sous le nom de «nubien du Kordofan» et localement appelé «ajang». Le plus connu est le dilling, parlé autour de la ville du même nom. Enfin, le midob et le birgid (presque éteint aujourd'hui) sont respectivement localisés dans les provinces du Darfour du Nord et du Darfour du Sud. 
- Le groupe taman se situe à cheval sur le Soudan et le Tchad et se compose de deux groupes dialectaux, le tama et le mararit, parlés par près de 150000 locuteurs.

- Enfin, le groupe nyima comprend deux parlers des Monts Nouba, le nyimang (dialectes ama et mandal) et l'afitti, avec près de 80000 locuteurs.

Parmi toutes ces langues, très peu disposent d'une description satisfaisante. C'est surtout pour les langues nubiennes qu'il existe de bonnes grammaires et des dictionnaires. Mais la situation s'améliore, en partie grâce aux projets linguistiques de l'université de Cologne et à ceux que j'ai personnellement conduits ou initiés.

La dispersion des langues Son entre l'Ennedi au Tchad et la vallée du Nil au Soudan est expliquée, comme nous l'avions évoqué dans notre premier chapitre, par la dessiccation d'un affluent du Nil, le Wadi Howar, entre le quatrième et le deuxième millénaire avant notre ère, obligeant les populations établies sur les rives à se réfugier dans des zones plus hospitalières à l'est, au sud et à l'ouest de la rivière disparue. Quant aux locuteurs du nara (ex-Baryas), isolés en Érythrée où ils sont attestés depuis la fin de l'Antiquité (ils sont cités dans la stèle d'Ézana dont le texte figure aux p.326-327, à la fin du chapitre précédent), ils ont très probablement transité par le Nil, puis l'Atbara, qui porte leur nom: cet affluent, aujourd'hui le dernier à rejoindre le fleuve, est connu dans les transcriptions des géographes alexandrins sous la forme Astaboras ou Astabaras, ce qui signifie «la rivière des Baryas" (méroïtique classique * ato Bres-o).

Toutes les langues Son ont conservé des structures très proches:

des origines

à la chute

du sultanat

Fung elles ne possèdent pas de genre grammatical, le verbe est à la fin de la phrase, l'adjectif suit le nom, le génitif précède le substantif qu'il détermine. Elles utilisent des postpositions et non des prépositions et recourent le plus souvent à des suffixes et non des préfixes. Dans le domaine morphologique, on trouve un article suffixé de forme ${ }^{*}-r$ ou ${ }^{*}-l$, soit opérationnel (méroïtique, taman), soit vestigiel (nubien, nara); une négation *ma (toutes les langues, sans lien génétique avec la négation arabe $m a$ ), une marque d'accusatif en ${ }^{*}-g$, ici encore opérationnelle (nubien, taman, nyimang) ou vestigielle (méroïtique, nara); un suffixe de pluriel pronominal en ${ }^{*}-g u$ (nubien, nara, taman) qui a en méroïtique évolué en - $b a$, par exemple dans l'article pluriel -leb, suivant un processus bien connu en linguistique, notamment dans les langues indo-européeennes (cf. gaulois bana, "femme», vs. grec gunê; roumain limbă, «langue», du latin lingua).

Dans le domaine lexical, la grande distance chronologique qui sépare ces langues a largement érodé le vocabulaire initial. Les traductions des termes les plus basiques du méroïtique sont, de plus, encore peu nombreuses. Toutefois, les correspondances sont édifiantes, particulièrement 
avec le nubien qui est le groupe génétiquement le plus proche. On citera les exemples suivants, où le proto-nubien est la langue nubienne originelle, reconstruite à partir des langues modernes et du vieux-nubien, et où le proto-Son désigne les formes reconstruites pour l'ensemble du soudanique oriental nord.

\begin{tabular}{|c|c|c|c|c|}
\hline \multicolumn{3}{|c|}{ méroïtique } & proto-Son & proto-nubien \\
\hline are- & {$[\mathrm{ar}]$} & «prendre» & *ar- & *aar- \\
\hline$k d i$ & {$[\mathrm{kadi}]$} & «femme» & ${ }^{*}$ kadi / *kari & ${ }^{*}$ kari \\
\hline$m s$ & {$[\mathrm{maca}]$} & «soleil» & $*^{*}$ masi & ${ }^{*}$ eesi $\left({ }^{*} \mathrm{~m}\right.$ initial amuii) \\
\hline wide & {$[\operatorname{wid}(\partial)]$} & «frère» & ${ }^{*}$ wer- & ${ }^{*}$ weri \\
\hline wle & [wal] & «chien» & ${ }^{*}$ wel & *wel \\
\hline
\end{tabular}

Il ne fait pas de doute que cette découverte récente va accélérer les progrès dans la compréhension de la langue méroïtique et la traduction des textes. Toutefois, la comparaison linguistique en ce domaine doit être conduite avec précaution. Les racines Son, en effet, sont souvent brèves et comportent un inventaire réduit de phonèmes, tant consonnes que voyelles, si bien que de nombreux homonymes existent: *ar- par exemple est la forme reconstruite pour "prendre», mais aussi pour «enfanter», «engendrer» (cf. méroïtique eri-ke). Il est probable que ces homonymes étaient souvent distingués par des tons, hauts ou bas, qui sont attestés dans toutes les langues Son modernes et devaient aussi exister en méroïtique, bien qu'ils ne soient pas notés dans l'écriture, comme plus tard en vieux-nubien. Pour toutes ces raisons, on devra continuer à encadrer l'usage de la comparaison linguistique par les méthodes traditionnelles fondées sur l'analyse contextuelle des textes et une bonne connaissance des réalités de la civilisation méroïtique.

\section{Les textes méroïtiques beaucoup d'épitaphes et peu de littérature}

Les quelque deux mille inscriptions actuellement retrouvées entre le sud de l'Égypte et le nord du Soudan ne peuvent se comparer, ni quantitativement, ni qualitativement, avec l'abondante documentation disponible en égyptien. Nous avons précédemment invoqué le moindre recours à l'écrit dans le royaume koushite, mais d'autres raisons expliquent cette situation. La population était moins nombreuse, de l'ordre de 900000 habitants pour Koush, contre 4 à 5 millions pour l'Égypte, bien que ces chiffres doivent être pris cum grano salis. D'autre part, les fouilles sont plus récentes et moins nombreuses au Soudan. Enfin, le climat soudanais, au moins sous la boucle du Nil, alterne des périodes de chaleur intense et des épisodes 


\section{Un exemple de texte méroïtique l'épitaphe de la dame Wiritélito}

Cette stèle funéraire a été découverte lors des fouilles que Woolley et RandallMacIver effectuèrent entre 1908 et 1910 sur la grande nécropole de Karanóg, en Basse-Nubie. La localité était en effet à l'époque tardive le siège des vicerois de Nubie, représentant le pouvoir central dans le nord du royaume. La stèle de Wiritélito a été retrouvée à l'avant de sa pyramide (G 217), dans les ruines de la chapelle funéraire. Elle se présente comme un bloc rectangulaire, muni d'un bec (apex) sur le côté supérieur, et entièrement couvert de signes. Le cadre gravé sur le champ de la stèle, divisé en douze lignes par des traits horizontaux, n’a pas suffi à placer l'ensemble de l'épitaphe prévue. Il a fallu recourir, comme il est usuel pour les tables d'offrandes mais exceptionnel pour les stèles, aux bords extérieurs, où la fin de l'inscription court dans le sens inverse des aiguilles d'une montre. Le style paléographique des signes indique une date dans la seconde moitié du $\mathrm{III}^{\mathrm{e}}$ siècle de notre ère.

Le texte avait été publié en 1911 par Griffith dans Karanóg. Il est enregistré au Répertoire d'épigraphie méroïtique sous le numéro REM O289. Nous en présentons ici une relecture et une traduction en petite partie

le Soudan

\section{6}

des origines

à la chute

du sultanat

Fung lacunaire, car le sens de certains titres et fonctions exercés par des parents de la défunte (senete, soni, atos, etc.) n'est pas entièrement clair. Les deux points transcrivent le séparateur de mots, dont l'usage n'est pas constant, les nombres en exposant correspondent à la numérotation des lignes puis des côtés de la stèle. Les contractions dues à la loi de Griffith (voir notre section grammaticale ci-dessus, p. 355 sq.) ont été développées pour que le lecteur puisse percevoir la composition des groupes nominaux: $k d i t o w i$, "elle était la sœur» (ligne 10), est ainsi transcrit kdise-l-owi, et kdisebetowi, «elle était leur sœur», (ligne 12) est transcrit kdise-bese-l-owi.

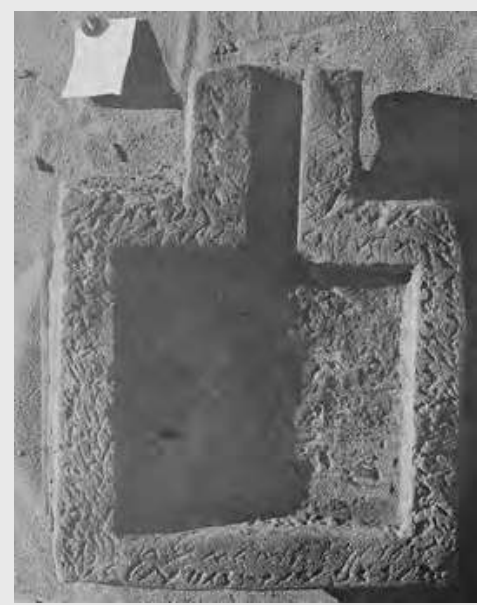

Table d'offrandes

de la dame Wiritélito

(orthographié Wirétélito)

Rem 0219, fonds Leclant.

d'Arététéli, fils

de la dame Wiritélito.

REM 0220, fonds Leclant.

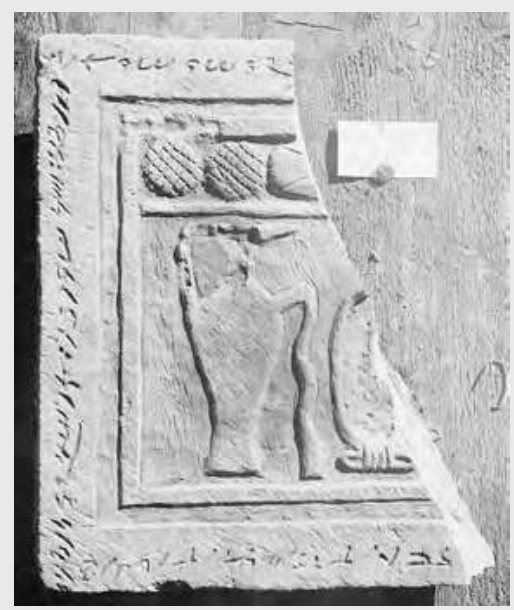




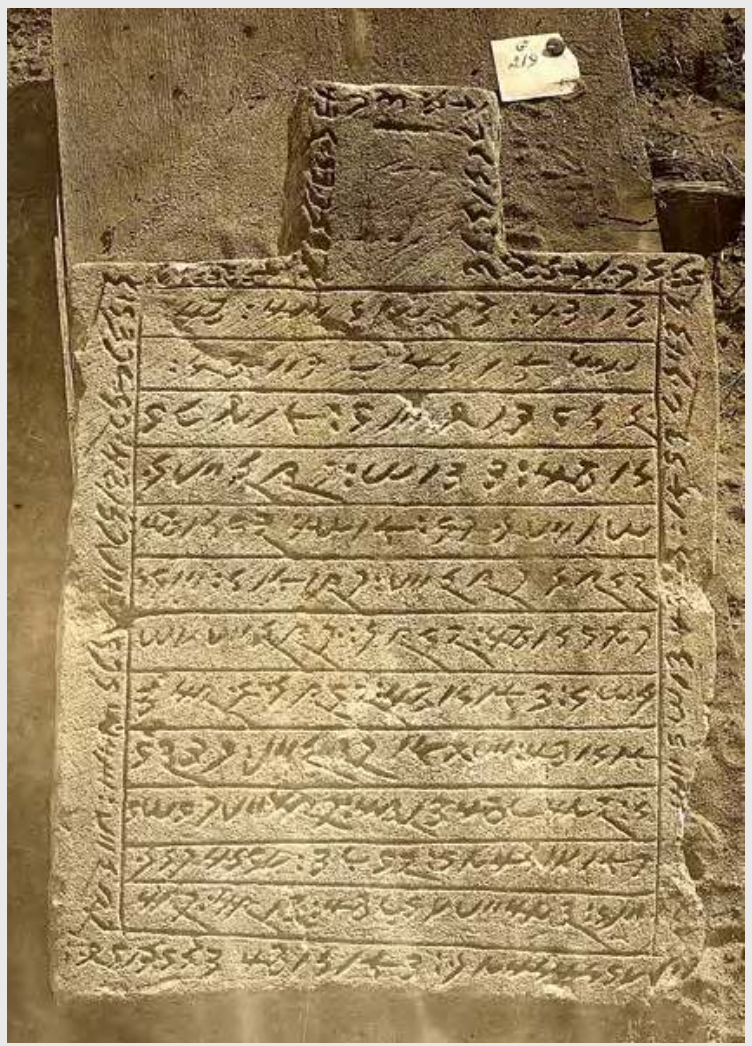

Stèle

de la dame Wiritelito, nécropole de Karanóg, seconde moitié du III siècle apr. J.-C., ReM 0289, fonds Leclant.

$46: 4 / 1 / \mathrm{sw} / 3: 43 / 6$ : $46 / 17 \div 4$ S15 $4 \mathrm{~W}$

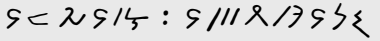
夕 VII $<ß 3: \omega / 33: \sigma / s^{4}$

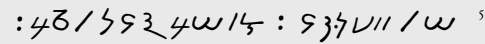

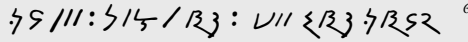

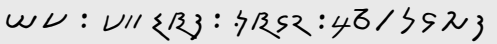

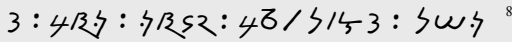

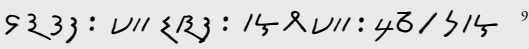

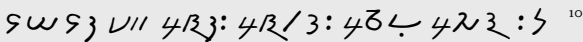

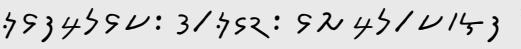

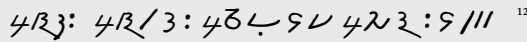

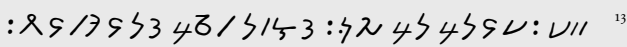

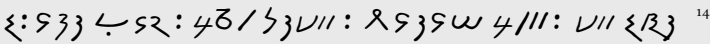
: 145353/3 $43 \leq: 533.52{ }^{16}$

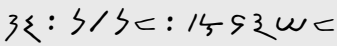
: S3S : 15 S३S ${ }^{18}$ III sw/3: $433^{11 / / 1 / 6: 15} 53<343\left\{{ }^{19}\right.$ 


$$
: 4 \sigma / 17 \div 4 \text { S/s 4W } 46: 4 / 1 / \text { sw/3:43/6 }
$$
${ }^{1}$ Wos- $i$ : Sorey-i:
Wi2 ritelito
q-owi:
Isis+ô!
Osiris+ô!
Wiritélito
celle-ci est;

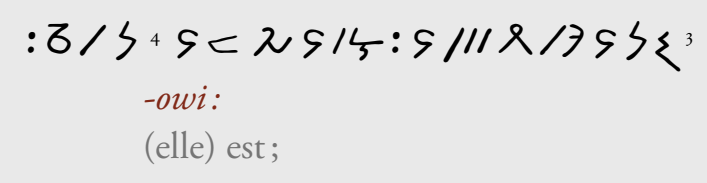

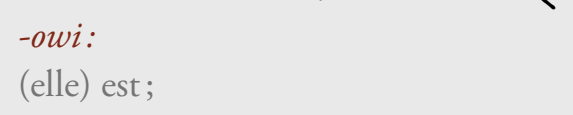
3Pleqneye: tedxe- $\quad 4-l$
de Baléqonéyé fille enfantée la (elle) est;

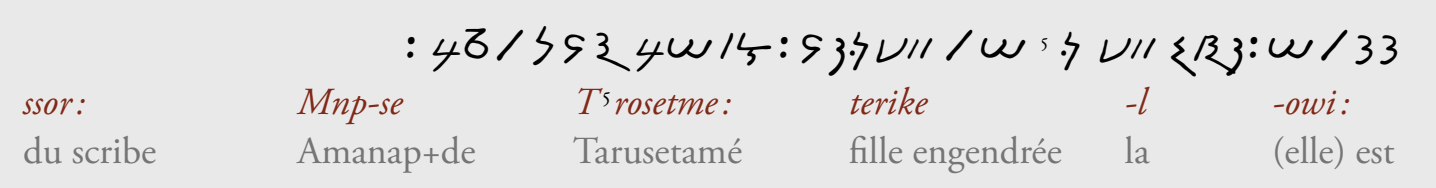

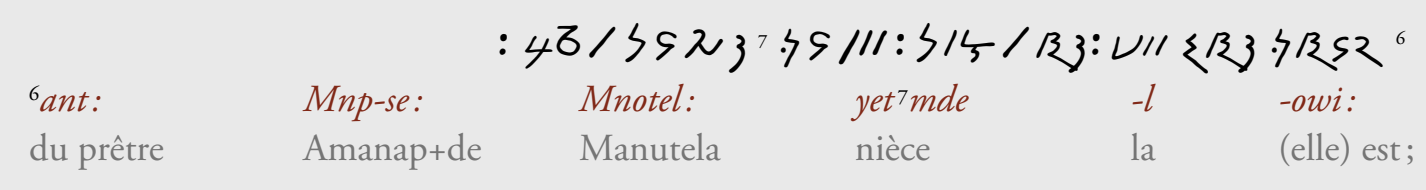

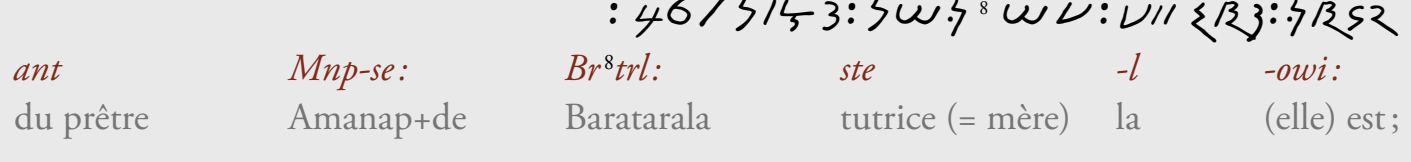

le Soudan

\section{8}

ant:

Tni:

s'te

$$
: 46 / 315 \text {, } 3: 4 ß 3: 3 ß \leqslant 2
$$

du prêtre

Tani

tutrice (= mère) la

-owi:

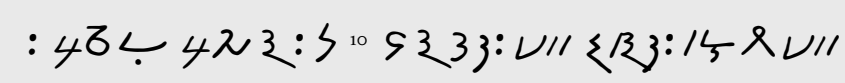

des origines

à la chute

du sultanat

Fung

senete:

Mnp-se:

Mske ${ }^{10}$ :

kdise

$-l$

-owi:

du senete (titre) Amanap+de Mashakéla sœur la (elle) est

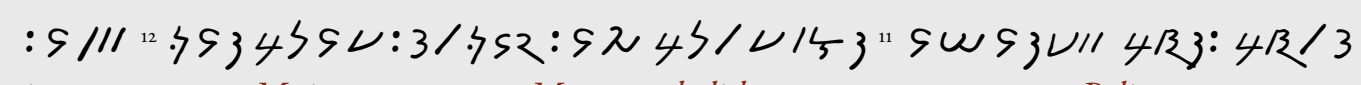

$\begin{array}{llll}\text { soni: } & \text { Mni-se } & \text { Mere }{ }^{11} \text { mtebolide: atos: } & \text { Belimet }{ }^{12} y e: \\ \text { du soni (titre) } & \text { Amon+de } & \text { Mérématébolidé atusha (titre) } & \text { Bélimétayé }\end{array}$

kdise

$-b e-s e$

sour

eux+de

$-l$

$: 46<5 レ 42 々$

(n)

la

-owi:

(elle) est;

soni:
du soni (titre)

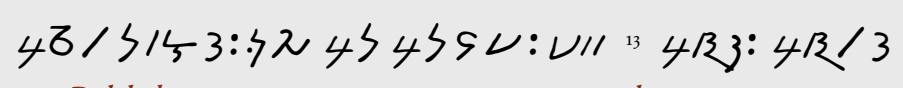

Mni ${ }^{13}$-se Belilidt: ste $-l \quad$-owi

Amon+de Bélilidata tutrice (=mère) la (elle) est;

sleqene:

${ }^{14} \mathrm{Mnp}$-se: $\quad$ Yiremene:

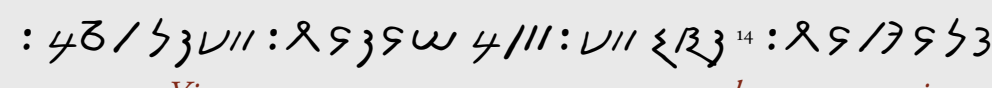

du shalqun (titre) Amanap+de Yirémène épouse la (elle) est; 
$x-$

repas

$x-$

repas

$W[o] s-i$

Isis+ô ! mhe:

abondante

mhe

abondant

mlo

bon

lh

grand

Sorey- $i$

Osiris+ô!
$: 145353 / 3^{15} \leq: 533<52$

$p^{15}$ so-he-kete:

faites qu'(elle) boive;

$p s i-{ }^{17} x r$-kete:

$: 1593 \omega \in{ }^{17} 43 \leqslant: 933$ לS2 ${ }^{16}$

faites qu'(elle) mange;

$-l$ :

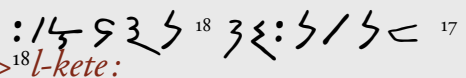

un $p-h<0>{ }^{18}$ l-kete:

faites que (lui) soit servi;

$-l$ :

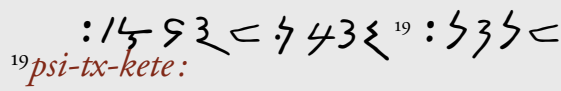

un

faites que (lui) soit offert;

/II sw/3:43\%/III

Ô Isis, ô Osiris! Voici Wiritélito.

Elle a été enfantée par Baléqonéyé; elle a été engendrée par le scribe d'Amanap (Amon de Napata) Tarusetamé.

Elle était la nièce de Manutéla, prêtre d'Amanap. Elle était la mère de Baratarala, prêtre d'Amanap. Elle était la mère du prêtre Tani. Elle était la sœur de Mashakéla, senete d'Amanap. Elle était la sœur de Mérématebolidé, soni d'Amon et de l'atusha Bélimétayé. Elle était la mère de Bélilidata, soni d'Amon. Elle était l'épouse de Yirémène, shalqun d'Amanap.

Faites qu'elle boive de l'eau en abondance! Faites qu'elle mange du pain en abondance. Faites-lui servir un bon repas! Faites-lui offrir un grand repas, ô Isis, ô Osiris!

Lépitaphe suit parfaitement le schéma prototypique des textes funéraires méroïtiques: invocation à Isis et Osiris, nomination de la défunte, puis de sa mère et de son père, longue description de sa situation sociale, en tant que nièce, mère, sœur et épouse de notables, bénédictions A (eau), B (pain) et $C$ («le bon repas»), auxquelles s'ajoute la rare bénédiction $\mathrm{D}$, celle du "grand repas", qui fait toutefois doublon avec la précédente. Le texte se termine par une reprise d'invocation assez fréquente. 
La dame Wiritélito appartient à la classe sacerdotale de la cité de Karanóg et provient d'une famille suffisamment aisée pour que ses membres les plus influents aient été inhumés sous des pyramides de briques de bonne taille ( $3,20 \mathrm{~m}$ d'arête pour celle de Wiritélito). Sa tombe a été pillée et la superstructure s'est effondrée, mais, outre la présente stèle, on a retrouvé à proximité une table d'offrandes à son nom, où il est cette fois orthographié Wirétélito (Rem 0219). Elle n'appartient pas toutefois à la classe dirigeante, ne citant aucun vice-roi (peseto) ou prince ( $p q r)$ dans sa lignée.

Deux autres épitaphes de sa famille sont connues: la table d'offrandes et la stèle (Rem O220 et O221) d'un de ses fils, Arététéli, prêtre d'Amanap, curieusement non cité ici, peut-être parce qu'il était trop jeune au décès de sa mère. La plupart des membres de cette famille appartient au personnel du temple local d'Amanap, c'est-à-dire Amon de Napata, bien qu'originellement le nom repose sur un jeu de mots entre l'égyptien $J m n n$ $J p . t$, "Amon de Louqsor", et Jmn (n) Npt, "Amon de Napata». Un oncle de Wiritélito, Manutéla, exerçait déjà la fonction de "prêtre d'Amanap". Un de ses frères a servi le même dieu, bien que son titre (senete) soit de sens obscur. Elle a épousé un "shalqun» (peut-être "chef de district») rattaché au même temple.

Au moins deux de leurs enfants (dont Arététéli, non mentionné ici) ont hérité du titre de "prêtre d'Amanap". L'épitaphe de Wiritélito est un des principaux documents qui atteste de la transmission des charges dans la lignée maternelle, ce qui constitue une rupture par rapport à la tradition égyptienne, où la lignée paternelle était privilégiée, les fils héritant

des origines

à la chute

du sultanat

Fung généralement des fonctions de leurs pères.

Stèle d'Arététéli,

fils de la dame Wiritélito. REM 0221, d'après Griffith, Karanóg, 1911.

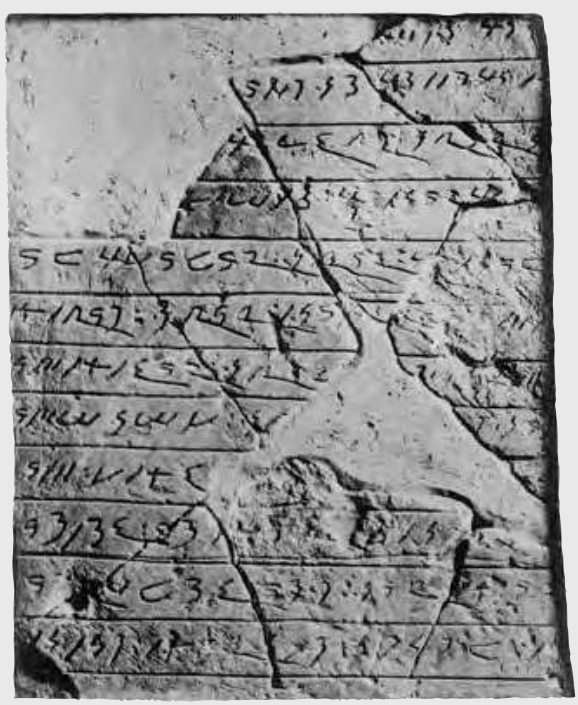


pluvieux parfois diluviens, ce qui est extrêmement dommageable à la conservation des éléments organiques. Or, l'évolution des signes cursifs méroïtiques au fil des siècles, avec des tracés moins anguleux et des queues de signes de plus en plus allongées, montre que les scribes ont adapté leurs graphies à une écriture effectuée au pinceau et non au ciseau des lapicides. Il faut en déduire que le papyrus était un support courant, quoique les seuls exemplaires de papyri méroïtiques connus aient été trouvés à Qasr Ibrim, en Nubie égyptienne, et récemment publiés par Jochen Hallof. Plus au sud, le matériau ne s'est probablement pas conservé, bien que nous ne soyons pas à l'abri d'heureuses surprises dans des fouilles à venir. Le support d'écriture le plus fréquemment retrouvé, parce que le mieux conservé, est évidemment la pierre, qu'il s'agisse de monuments funéraires ou de murs de temples. On possède aussi de nombreux ostraca, tessons de céramiques qui fournissaient un support bon marché, où les textes sont généralement tracés au pinceau. Des inscriptions sur cuir et sur métal ont aussi été découvertes, en nombre plus réduit.

Les épitaphes, il est vrai souvent longues et détaillées, constituent près de $40 \%$ du total des textes. Elles se présentent sur deux types de monuments, les stèles funéraires et les tables d'offrandes. Sur ces dernières, l'inscription court généralement le long des bords, le centre étant consacré à la représentation des offrandes, pains ronds, aiguières d'où s'écoule un filet d'eau, fleurs de lotus, etc. Elles suivent le plus souvent un schéma constant: invocation à Isis et Osiris, nomination du défunt, filiation maternelle et paternelle, description sociale de l'individu par l'énoncé de ses fonctions et de ses liens familiaux avec des parents prestigieux dont les titres sont donnés, bénédictions finales généralement au nombre de trois, celle garantissant de l'eau au défunt (formule A), celle du pain (formule B) et celle du «bon repas» (formule C). La partie descriptive est évidemment plus ou moins développée selon le rang et la famille de l'individu: elle est souvent une source d'informations précieuses sur la société provinciale du royaume koushite. Curieusement, elle est absente dans le Sud, par exemple à Méroé, pourtant siège du pouvoir central, où les épitaphes, y compris celles des souverains, sont extrêmement laconiques. Les inscriptions funéraires sont les mieux comprises parmi les textes méroïtiques, en raison de leur contenu stéréotypé et d'une focalisation sur elles de la recherche depuis l'époque de Griffith.

Une autre catégorie de textes, historiquement importante mais peu nombreuse, est celle des inscriptions royales. Généralement gravées sur des stèles de grès ou de granite, plus rarement sur des murs de temples, elles sont au nombre d'une vingtaine. Les plus célèbres sont celles du roi Tanéyidamani, datant du milieu du II $^{\mathrm{e}}$ siècle av. J.-C., aujourd'hui 
le Soudan

372

des origines

à la chute

du sultanat

Fung à Boston, celle de la Candace Amanirénas et du prince Akinidad, peu avant le début de notre ère, aujourd'hui conservée à Londres, et l'inscription murale du roi blemmye Kharamadoyé, au début du ve siècle, gravée dans le temple de Kalabcha en Égypte. Elles poursuivent en méroïtique la tradition napatéenne des chroniques de règne rédigées en égyptien, avec quelques différences notables: elles ne comportent pas de repères chronologiques par années régnales par exemple. Leur contenu semble très similaire: après un protocole initial détaillant les titres du souverain et un éloge de sa personne, se développe une série de narrations où alternent les actes de piété envers les dieux et les campagnes militaires contre les rebelles ou les peuples ennemis. Il s'en faut de beaucoup que ces textes, dont le vocabulaire et la morphologie riches défient notre faible connaissance de la langue, soient aussi bien compris que les épitaphes.

Le corpus des textes religieux gravés dans les temples, où ils légendent les représentations des souverains et des dieux, a la particularité d'être entièrement rédigé en méroïtique hiéroglyphique, alors que les autres types d'inscriptions sont presque tous en cursive. La plupart d'entre eux, que ce soit à Naga, Méroé, Dangeil, Barkal ou Amara, datent du règne conjoint de la Candace Amanitoré et du roi Natakamani, vers 60 apr. J.-C. Les temples comportent aussi très souvent des graffiti pieux en cursive, appelés "proscynèmes", tracés par les pèlerins dans les parties de ces monuments ouvertes aux profanes. Le temple d'Isis à Philae en est particulièrement riche, mais on en trouve aussi beaucoup à Kawa, Méroé et el-Hassa. Un type de graffiti assez commun accompagne la représentation d'un pied ou deux, parfois chaussés de sandales: le texte, connu aussi en démotique et en grec, informe le visiteur que "ce pied qui appartient à Untel a été gravé en présence d'Isis» (ou d'un autre dieu). Il témoigne que le pèlerin est bien venu en personne adorer la divinité.

Un autre type de texte religieux, délivré par le clergé des temples contre rémunération, assure une protection divine contre les malheurs de la vie. Ce sont les «décrets oraculaires amulétiques», qui perpétuent dans le royaume de Méroé une tradition attestée en Égypte au début du premier millénaire avant notre ère. Après une introduction codifiée, ils énumèrent les dangers auxquels, par décret divin obtenu par oracle, échapperont leurs bénéficiaires, bien que cette partie du texte nous soit incompréhensible et que seuls les parallèles égyptiens nous permettent de l'affirmer. Plusieurs d'entre eux sont attestés sur les papyri retrouvés à Qasr Ibrim, d'autres ont été recopiés sur des ostraca, conservés dans les maisons de particuliers, ou même sur des bandelettes de cuir que l'on nouait sur le bras en guise de talisman. Une coutume similaire s'est conservée en Afrique, notamment en terre d'Islam, des sourates du Coran ayant remplacé les décrets divins. 
Les textes utilitaires étaient inscrits sur des tessons de poterie ou ostraca: on a retrouvé ainsi des comptes administratifs ou commerciaux qui sont notre principale source sur le système de numération méroïtique. De courtes inscriptions tracées sur des objets, majoritairement des vases en contexte funéraire, nous sont aussi parvenues. Elles indiquent le nom et le titre du dédicateur de ces offrandes ou, dans d'autres cas, le contenu. Une des rares inscriptions bilingues provient ainsi de jarres retrouvées brisées dans les chambres funéraires de la reine Nahirqo à Méroé (pyramide Beg. N.11). Elle précise en démotique égyptien le contenu: jrp n Kmy, "vin d'Égypte», et est paraphrasée en méroïtique: Qomo-s-o, "cela vient d'Égypte». Avec un texte aussi succinct, on est évidemment bien loin de la Pierre de Rosette!
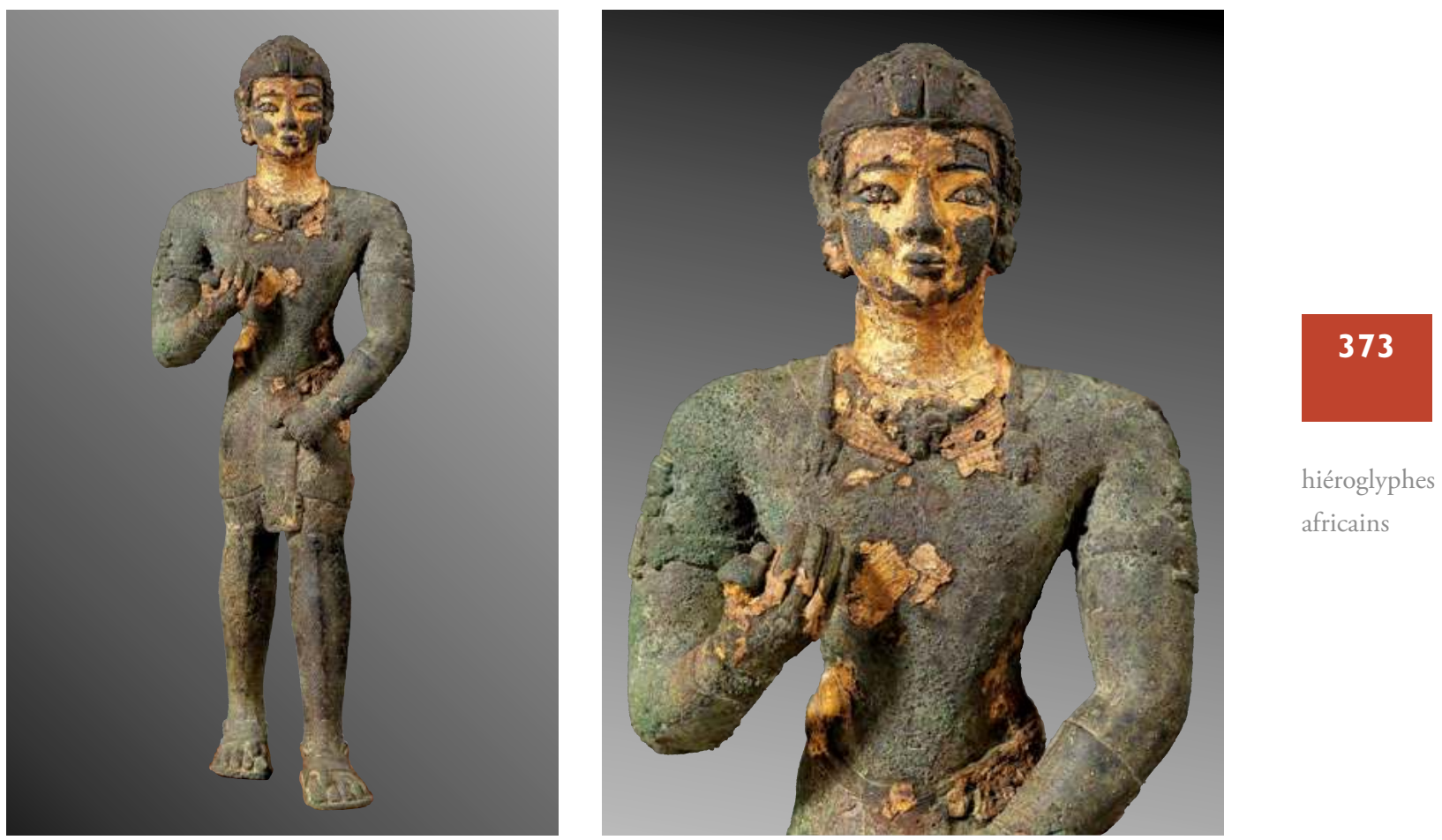

hiéroglyphes africains

Le roi archer. Tabo, île d'Argo, cour du grand temple. Sculpture méroïtique datant soit du III ${ }^{\mathrm{e}}$ siècle av. J.-C., soit, plus probablement, du règne de Natakamani ( $\mathrm{I}^{\mathrm{er}}$ siècle de notre ère) : ce dernier ayant construit à Tabo, cette seconde hypothèse paraît la plus plausible. Khartoum, musée national du Soudan, SNM 24705. 